\title{
A local constitutive model with anisotropy for various homogeneous 2D biaxial deformation modes
}

\author{
S. Luding and E. S. Perdahciog̃lu \\ Multi Scale Mechanics (MSM), TS, CTW, UTwente, \\ POBox 217, 7500 AE Enschede, Netherlands; \\ s.luding@utwente.nl
}

submitted: 19.10.2010 - revised: 20.01.2011

\begin{abstract}
A local constitutive model for granular materials with anisotropy is proposed and applied to different biaxial box deformation modes. The simplified version of the model (in the coordinate system of the biaxial box) involves only scalar values for hydrostatic and shear stresses, for the isotropic and shear strains as well as the new parameter, the (scalar) anisotropy modulus.

A non-linear constitutive evolution equation, for both shear stress and anisotropy, during deviatoric (shear) deformation is based on observations gained from Discrete Element Method (DEM) simulations. While parameters like the bulk modulus are set to constant, for the sake of simplicity, the model involves a yield stress and a maximal anisotropy as well as the corresponding deviatoric shear-rate pre-factors for incremental stress and incremental anisotropy modulus.

In this study, the self-consistency of the simple-most model is discussed before it is applied to various bi-axial deformation modes. Constant anisotropy is compared to evolving anisotropy, where the sign accounts for the direction (tension or compression corresponds to positive or negative strain, respectively). Generalization to arbitrary orientation and possible non-coaxial strain, stress and fabric tensors is not yet attempted in this study.
\end{abstract}

\section{Introduction}

Dense granular materials show peculiar mechanical properties quite different from classical fluids or solids $[1,2]$. These phenomena involve dilatancy, yield stress and history dependence - among many others. The reason is the inherent discrete structure of particulate packings that is far from understood [3-11]. Particularly, if a granular packing is subject to isotropic compression the shear stress remains close to zero and the major control parameters are the volume fraction and the pressure. However, under shear deformation, not only shear stress builds up until it reaches a yield-limit, but also the anisotropy of the contact network develops, as related to the creation and destruction of contacts and force-chains, see e.g. [12-16] and also Ref. [9] and references therein. These phenomena are at the origin of the interesting behavior of granular media, in particular their "memory", i.e., their history dependence The contact structure, its evolution and especially its anisotropy are neglected in many continuum models of particulate matter.

In order to better understand and model the deformation behavior of particle systems, the Discrete Element Method is a very useful tool [7,11,16-18]. From the analysis of such simulations, when using consistent averaging procedures [18-24], useful relations can be derived between the fabric, strain and stress tensors. The collective behavior of the particles can then be described by a homogenized model, using macroscopic definitions and formulations of these tensors. The homogenized model has advantages since it can be implemented in a FEM software to observe the deformation mechanisms in arbitrary (inhomogeneous) loading conditions. 
Most of the existing models describe the constitutive behavior of granular materials but one important ingredient missing is the anisotropy and possible non-coaxiality of the macroscopic tensors. Only few theories, see e.g. $[11,16,18,25-27]$ and references therein, involve an anisotropy state variable or non-coaxiality; even less deal with both anisotropy and rotations [24,28-32]. The DEM simulations reveal that considerable anisotropy develops in the system during deformation. This is an essential part of the constitutive model because it contains the information how the different modes of deformation have affected the mechanical state of the system during the deformation. In this sense, the anisotropy is a history parameter.

In the following, a constitutive model that is based on observations from DEM simulations will be presented in its simplest form. Anisotropy is included in the model through the evolution of the stiffness tensor components in a prescribed manner. The (classical) isotropic and shear moduli are set constant, at first, in order to be able to focus on the effect of anisotropy exclusively.

\section{Model System and Theory}

The model system is shown schematically in Fig. 1 as used for the DEM simulations in Ref. [18]. In this system, for small deformations, the strain $\varepsilon_{22}$ in the $y$ direction and the normal stress $t=\sigma_{11}$ in the $x$ direction are prescribed. (Below we refer to this bi-axial, side-stress controlled deformation as mode 3). All bi-axial deformation modes, as specified below in Tab. 1, can be described by a strain tensor (incremental) with only diagonal components in the global coordinate system:

$$
[\varepsilon]=\left[\begin{array}{cc}
\varepsilon_{11} & 0 \\
0 & \varepsilon_{22}
\end{array}\right]
$$

which, naturally, are the eigenvalues of the strain tensor. Throughout this paper, small strain increments are implied, as denoted by $\delta \varepsilon$, if not stated explicitly otherwise. Since the decomposition of tensors into isotropic and deviatoric parts does not rely on "small", the $\delta$ is not used in this section.

The coordinate system of the box is the natural choice and identical to the tensor-eigensystem, and thus identical to the bi-axial system walls, if there is no wall friction involved.

The stress that develops in the system also consists of the diagonal terms only (with components given in the global coordinate system):

$$
[\boldsymbol{\sigma}]=\left[\begin{array}{cc}
\sigma_{11} & 0 \\
0 & \sigma_{22}
\end{array}\right] .
$$

Initially, an isotropic stress is applied to the system to confine the particles. The initial strain and stress conditions therefore are given as:

$$
\left[\boldsymbol{\varepsilon}_{0}\right]=\left[\begin{array}{ll}
0 & 0 \\
0 & 0
\end{array}\right], \quad\left[\boldsymbol{\sigma}_{0}\right]=\left[\begin{array}{cc}
\sigma_{0} & 0 \\
0 & \sigma_{0}
\end{array}\right]
$$

where $\sigma_{0}$ is the initial (isotropic) hydrostatic stress applied.

One very important point for the model is the selection of a sign convention for strain and stress. In the present work, the general convention is positive $(+)$ for dilatation, extension and negative (-) for compression, contraction. According to this definition, when the top boundary is moved inwards/downwards $\varepsilon_{22}$ (the prescribed strain) will have a negative value whereas $\varepsilon_{11}$, moving outwards, will be positive.

The strain can be decomposed into an isotropic part and a deviatoric part:

$$
\varepsilon=\varepsilon^{V}+\varepsilon^{D}
$$




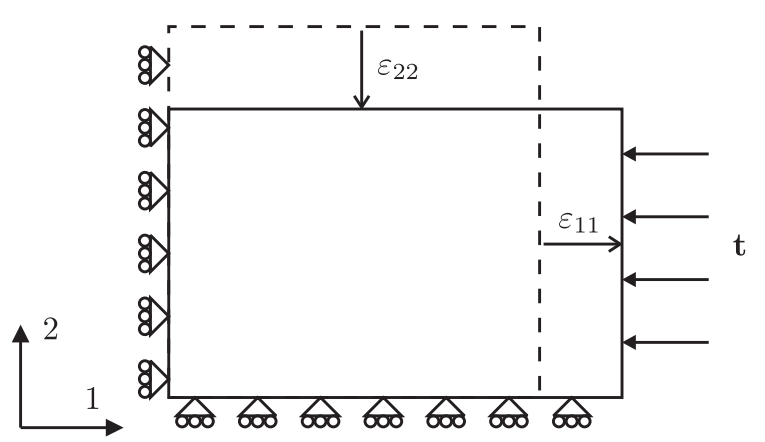

Figure 1: Illustration of the bi-axial model system used for the DEM simulations. The vertical displacement $\varepsilon_{22}(t)$ and the horizontal stress (traction) $\sigma_{11}=t=\sigma_{0}$ are prescribed.

where capital letter superscripts are used for tensors, with the isotropic part given as

$$
\varepsilon^{V}=\frac{\varepsilon_{11}+\varepsilon_{22}}{2}\left[\begin{array}{ll}
1 & 0 \\
0 & 1
\end{array}\right]=\varepsilon^{v} \mathbf{I}=\frac{1}{\mathcal{D}} \operatorname{tr}(\varepsilon) \mathbf{I},
$$

with dimension $\mathcal{D}=2$, so that the volume change is $\operatorname{tr}(\varepsilon)=2 \varepsilon^{v}$ in the following ${ }^{1}$. (Note that the abbreviation $\operatorname{tr}(\varepsilon)=\varepsilon^{\mathrm{vol}}$ is used in many other studies, however, we adopt the alternative definition that involves the division by dimension - similar to the definition of the pressure (isotropic stress $\sigma^{h}$ ) below). Accordingly the deviatoric part is:

$$
\varepsilon^{D}=\varepsilon-\varepsilon^{V}=\left[\begin{array}{cc}
\frac{\varepsilon_{11}-\varepsilon_{22}}{2} & 0 \\
0 & \frac{\varepsilon_{22}-\varepsilon_{11}}{2}
\end{array}\right]=\frac{\varepsilon_{11}-\varepsilon_{22}}{2}\left[\begin{array}{cc}
1 & 0 \\
0 & -1
\end{array}\right]=\gamma \mathbf{I}^{D},
$$

where $\gamma$ is the scalar that describes the pure shear deformation as $\gamma=\frac{\varepsilon_{11}-\varepsilon_{22}}{2}$ and $\mathbf{I}^{D}$ is the traceless unit-deviator in $2 \mathrm{D}$. The unit-deviator has the eigenvalues, +1 and -1 , with the eigendirections $\hat{n}(+1)=\hat{x}$ and $\hat{n}(-1)=\hat{y}$, where the hats denote unit vectors.

As seen in Eq. (5) the isotropic part can be expressed fully using only one scalar, independent of the coordinate system chosen. This defines the isotropic strain and hence the amount of volume change. Positive and negative $2 \varepsilon^{v}$ correspond to volume increase and decrease, respectively.

The same type of interpretation is not possible for the deviatoric part in general. According to the coordinate system that is chosen, the sign of the shear deformation changes, e.g., when the $x$ and $y$ directions are exchanged. Equivalently, when the deviatoric (shear) strain $\gamma$ changes sign, the deformation is reversed. An additional point concerning the strain terms is therefore the selection of the coordinate system and the type of deformation. If the coordinate system chosen is rotated from the biaxial orientation or the prescribed deformation is different from Eq. (1), one has offdiagonal terms, as discussed below. In order to keep the model as simple as possible, we restrict ourselves to biaxial deformations and the biaxial orientation of the coordinate system.

\subsection{Alternative formulation for arbitrary orientation}

To further elaborate this issue, we compare the two possible representations of the deviatoric part of a symmetric tensor. The first one is to define a positive scalar and let an orientation tensor completely take care of the direction:

$$
\varepsilon^{D}=\varepsilon^{d} \mathbf{D}\left(\phi_{\varepsilon}\right), \quad \text { with : } \varepsilon^{d}=\left|\frac{\varepsilon_{11}-\varepsilon_{22}}{2}\right|=|\gamma|,
$$

and the oriented unit-deviator:

$$
\mathbf{D}\left(\phi_{\varepsilon}\right)=\mathbf{R}\left(\phi_{\varepsilon}\right) \cdot \mathbf{I}^{D} \cdot \mathbf{R}^{T}\left(\phi_{\varepsilon}\right) \text {. with (clockwise) rotation matrix : } \mathbf{R}(\phi)=\left[\begin{array}{cc}
\cos \phi & -\sin \phi \\
\sin \phi & \cos \phi
\end{array}\right],
$$

\footnotetext{
${ }^{1}$ The extension of the model to three dimensions is in progress and will not change its spirit. It will be published in future studies - for a rough sketch of what this involves, see the conclusion section.
} 
and the angle between the horizontal and the positive eigen-direction of the deviator: $\phi_{\varepsilon} \in[0, \pi[$ (due to symmetry, $\phi_{\varepsilon}+\pi=\phi_{\varepsilon}$ ).

In the second case, which is not generally applicable, one allows the sign of the scalar value $\gamma$ to be positive or negative and brackets the orientation angles:

$$
\varepsilon^{D}=\gamma \mathbf{D}\left(\varphi_{\varepsilon}\right), \quad \text { with }: \quad \gamma=\frac{\varepsilon_{11}-\varepsilon_{22}}{2},
$$

where

$$
\mathbf{D}\left(\varphi_{\varepsilon}\right)=\mathbf{R}\left(\varphi_{\varepsilon}\right) \cdot \mathbf{I}^{D} \cdot \mathbf{R}^{T}\left(\varphi_{\varepsilon}\right) .
$$

and $\varphi_{\varepsilon} \in\left[0, \frac{\pi}{2}\left[\right.\right.$, since $\varphi_{\varepsilon}+\frac{\pi}{2}=\varphi_{\varepsilon}$ but $\gamma\left(\varphi_{\varepsilon}+\frac{\pi}{2}\right)=-\gamma\left(\varphi_{\varepsilon}\right)$.

Thus, in the general case, the magnitude of shear is represented by the positive scalar $\varepsilon^{d}>0$ and the orientation by $\mathbf{D}\left(\phi_{\varepsilon}\right)$. A reversal of strain direction then is indicated by a transition of $\phi_{\varepsilon} \rightarrow \phi_{\varepsilon} \pm \pi / 2$. However, in the biaxial system, it is possible to use $\gamma$ and distinguish between the two possible directions $\phi_{\varepsilon}=0$ and $\phi_{\varepsilon}=\frac{\pi}{2}$ by the sign of $\gamma$. This latter convention is adopted below, since the eigen-system does not rotate.

With the selection of the deformation and the coordinate system as described above, it is possible to define a rotation, which makes it possible to represent the shear strain with one scalar only. Since simple shear takes place at 45 degrees relative to the principal basis, one has:

$$
\varepsilon^{S}=\mathbf{R}\left(\frac{\pi}{4}\right) \cdot \varepsilon^{D} \cdot \mathbf{R}^{\mathrm{T}}\left(\frac{\pi}{4}\right)
$$

which gives:

$$
\varepsilon^{S}=\left[\begin{array}{cc}
0 & \frac{\varepsilon_{11}-\varepsilon_{22}}{2} \\
\frac{\varepsilon_{11}-\varepsilon_{22}}{2} & 0
\end{array}\right]=\gamma \mathbf{D}\left(\frac{\pi}{4}\right) .
$$

Eq. (12) shows that shear strain deformations (for the biaxial box geometry) can also be described with one scalar $\gamma$, since the orientation is hidden in the directed unit-deviator.

The same definitions can be applied to the stress tensor as well. The decomposition in this case is for the hydrostatic stress and the deviatoric stress:

$$
\boldsymbol{\sigma}=\boldsymbol{\sigma}^{H}+\boldsymbol{\sigma}^{D} .
$$

The hydrostatic part is given as

$$
\boldsymbol{\sigma}^{H}=\frac{\sigma_{11}+\sigma_{22}}{2}\left[\begin{array}{ll}
1 & 0 \\
0 & 1
\end{array}\right]=\sigma^{h} \mathbf{I}=\frac{1}{\mathcal{D}} \operatorname{tr}(\boldsymbol{\sigma}) \mathbf{I} .
$$

and, accordingly, the deviatoric part becomes:

$$
\boldsymbol{\sigma}^{D}=\boldsymbol{\sigma}-\boldsymbol{\sigma}^{H}=\left[\begin{array}{cc}
\frac{\sigma_{11}-\sigma_{22}}{2} & 0 \\
0 & \frac{\sigma_{22}-\sigma_{11}}{2}
\end{array}\right]=\sigma^{d} \mathbf{D}\left(\phi_{\sigma}\right)=\tau \mathbf{I}^{D},
$$

with $\tau=\frac{\sigma_{11}-\sigma_{22}}{2}$.

In this case when the same rotation as above is performed, one obtains the magnitude of the shear stress:

$$
\boldsymbol{\sigma}^{S}=\mathbf{R}\left(\frac{\pi}{4}\right) \cdot \boldsymbol{\sigma}^{D} \cdot \mathbf{R}^{\mathrm{T}}\left(\frac{\pi}{4}\right)=\left[\begin{array}{cc}
0 & \frac{\sigma_{11}-\sigma_{22}}{2} \\
\frac{\sigma_{11}-\sigma_{22}}{2} & 0
\end{array}\right]=\tau \mathbf{D}\left(\frac{\pi}{4}\right),
$$

where, according to their definition, both $\gamma$ and $\tau$ can be positive or negative.

\subsection{The simplest anisotropy model in $2 \mathrm{D}$}

Finally, the simplest additional tensor (that will be used for the calculations in the next section) is the anisotropy modulus tensor, $\mathbf{a}^{D}$ (second order), which is trace-less and thus described completely 
by its magnitude $A$ and its orientation $\phi_{A}$. Since we assume in the following that the orientation of anisotropy is co-axial with stress and strain in the bi-axial system, the only one new free parameter that remains is $A$, so that:

$$
\mathbf{a}^{D}=A \mathbf{I}^{D}, \quad \text { with : } A=\frac{C_{11}-C_{22}}{2},
$$

where $C_{11}$ and $C_{22}$ are defined below.

In summary, for the biaxial system, the tensors $\varepsilon^{D}, \boldsymbol{\sigma}^{D}$, and $\mathbf{a}^{D}$ can be represented by the scalars $\gamma, \tau$, and $A$, respectively, while the orientation is fixed to $\mathbf{I}^{D}$. Change of sign corresponds to reversal of deformation, stress, or anisotropy, respectively.

\section{Governing equations}

As in a general constitutive relation of anisotropic elasticity, the relation between stress and strain increments is:

$$
\delta \boldsymbol{\sigma}=\mathcal{C}: \delta \varepsilon+\delta \boldsymbol{\sigma}^{\text {struct }}
$$

where $\mathcal{C}$ is the (rank four) stiffness tensor of the system and $\delta \boldsymbol{\sigma}^{\text {struct }}$ is the stress change due to structural changes (leading to a change of $\mathcal{C}$ ) and sliding contacts, but will not be specified here and set to $\delta \boldsymbol{\sigma}^{\text {struct }}=0$ for the sake of simplicity. ${ }^{2}$ Note that the two terms in Eq. (18), and especially the non-linearity of the second, see subsection 4.2.6, can be seen as similar to the two terms in hypoplastic type theories, see Eq. (9) in Ref. [30] and references therein, or Eq. (1) in Ref. [33, 34], where Granular Solid Hydrodynamics (GSH) is concerned. Furthermore, the split between elastic and plastic strain and stress $[16,33-35]$ is a related issue, but will not be detailed in this study.

\subsection{Nomenclature in the elastic limit}

Since in the biaxial box we deal with two components of stress and strain only, the following reduction can be performed:

$$
\left[\begin{array}{l}
\delta \sigma_{11} \\
\delta \sigma_{22}
\end{array}\right]=\left[\begin{array}{ll}
C_{1111} & C_{1122} \\
C_{2211} & C_{222}
\end{array}\right] \cdot\left[\begin{array}{l}
\delta \varepsilon_{11} \\
\delta \varepsilon_{22}
\end{array}\right]=\mathbf{C} \cdot\left[\begin{array}{l}
\delta \varepsilon_{11} \\
\delta \varepsilon_{22}
\end{array}\right]
$$

For simplicity in notation, the components of the stiffness will be referred to with reduced indices according to Eq. (19), i.e., $C_{11} \equiv C_{1111}, C_{22} \equiv C_{2222}$, and $C_{12} \equiv C_{1122}=C_{21} \equiv C_{2211}$.

As shown in the previous section, the incremental evolution of the hydrostatic stress is then given as a scalar equation using Eq. (19):

$$
\delta \sigma^{h}=\frac{\delta \sigma_{11}+\delta \sigma_{22}}{2}=\frac{C_{11}+C_{22}+2 C_{12}}{2} \delta \varepsilon^{v}+\frac{C_{11}-C_{22}}{2} \delta \gamma
$$

Similarly, the equation for incremental shear stress evolution is:

$$
\delta \tau=\frac{\delta \sigma_{11}-\delta \sigma_{22}}{2}=\frac{C_{11}-C_{22}}{2} \delta \varepsilon^{v}+\frac{C_{11}+C_{22}-2 C_{12}}{2} \delta \gamma
$$

These equations serve as the basic governing equations of the model. As abbreviations, in order to achieve the simplest version, the following scalars (with units of moduli) are defined [18, 22]:

$$
B=\frac{C_{11}+C_{22}+2 \lambda}{4}, \quad G=B-\lambda, \quad \text { with } \lambda=C_{12}, \quad \text { and } \quad A=\frac{C_{11}-C_{22}}{2},
$$

${ }^{2}$ This is at the origin of the different moduli for tension and compression strain as observed in real tests, which will enter the model via $A$ and $S$ below. Structural changes involve opening and closing of contacts, but also large-scale reorganizations of the particles, while the transition from sticking to sliding is due to finite coefficients of friction at the contact level, which is not considered here and will be discussed elsewhere. 
where $G$ that represents the bi-axial response to pure shear, corresponding to one of the Lamé constants, while $\lambda$ is the other Lamé constant. From the equations it is also apparent that $B$ is the bulk modulus that relates pressure and volume change, $\delta p=\delta \sigma^{h}=B \operatorname{tr}(\delta \varepsilon)=2 B \delta \varepsilon^{v}$, due to Eq. (5) and our sign convention, while $G$ is the shear modulus that relates the (pure, bi-axial) shear stress to shear strain, $\delta \tau=2 G \delta \gamma .{ }^{3}$ Finally, $A$ is the anisotropy modulus that couples bulk stress to shear strain and shear stress to isotropic strain, so that:

$$
\delta \sigma^{h}=2 B \delta \varepsilon^{v}+A \delta \gamma .
$$

Similarly, the equation for incremental shear stress evolution is:

$$
\delta \tau=A \delta \varepsilon^{v}+2 G \delta \gamma
$$

They can be used in different ways utilizing the anisotropy or non-linearity in the formulations, which will be discussed below. In all cases assumed here, however, one should note that $C_{12}=C_{21}$ holds, so that a single anisotropy modulus $A$ is the only new parameter when compared to isotropic materials.

\subsection{Model without anisotropy}

For the system to be isotropic, the stiffness in all directions should be the same. Hence, in the isotropic system $C_{11}=C_{22}$ must hold, so that $A=0$. When anisotropy starts developing, this equivalence condition is lost, as discussed in the following subsection.

In the isotropic case, the second term in Eq. (23) and the first term in Eq. (24) vanish and one arrives at the equations:

$$
\begin{aligned}
\delta \sigma^{h} & =2 B \delta \varepsilon^{v} \\
\delta \tau & =2 G \delta \gamma
\end{aligned}
$$

It is clear, in this case, that the hydrostatic stress only depends on the isotropic strain and similarly the shear stress only depends on the shear strain. For constant $B$ and $G$, this special case is thus purely linear with isotropic and deviatoric components decoupled.

Note that both $B$ and $G$ will develop with respectively applied isotropic or deviatoric (shear) deformations. But since this is not the focus of this study, we only use constant $B$ and $G$ in the following.

\subsection{Model with evolution of anisotropy}

When there is anisotropy in the system, i.e., $C_{11} \neq C_{22}$, the complete version of Eqs. (23) and (24) must be used. Even though $A$ is defined as the scalar anisotropy modulus, in the general case, it is actually a tensorial quantity with a certain orientation. However, in the biaxial system, similar to the strain and stress, it can be reduced to a scalar quantity, which can have both positive and negative values. Positive in our convention means that the horizontal stiffness is larger than the vertical one, and negative implies the opposite.

From Discrete Element Method simulations [18] of an initially isotropic system, with $A_{0}=0$, during deformation, anisotropy builds up to a limit $A^{\max }$. This a-posteriori rectifies the assumption of constant $B$ and $G$, to first order, since these two quantities change much less than $A$ for mode 3 deformations, as discussed below. It is also observed that the anisotropy develops only due to shear strain and not due to isotropic strain. Therefore, the evolution of $A$ can be described as:

$$
\frac{\partial A}{\partial \gamma}=-\beta_{A} \operatorname{sign}\left(A^{\max }\right)\left(A^{\max }-A\right), \quad \frac{\partial A}{\partial \varepsilon^{v}}=0
$$

\footnotetext{
${ }^{3}$ Note that we define $\gamma$ according to Eq. (6), i.e., the convention of defining $\gamma_{x y}=2 \varepsilon_{x y}$ is not applied here.
} 
with $A^{\max }=-\left|A^{\max }\right| \gamma /|\gamma|=-A^{m} \gamma /|\gamma|=-A^{m} \operatorname{sign}(\gamma)$, with positive maximal anisotropy, $A^{m}$, i.e., the sign of $A^{\max }$ is determined by the direction of shear. ${ }^{4}$ Note that Eq. (27) can also be written as

or

$$
\frac{\partial A}{\partial|\gamma|}=\beta_{A}\left(A^{\max }-A\right),
$$

$$
\frac{\partial A}{\partial \gamma}=\beta_{A} \operatorname{sign}(\gamma)\left(A^{\max }-A\right)=\beta_{A}\left(-A^{m}-\operatorname{sign}(\gamma) A\right)=-\beta_{A}\left(A^{m}+\operatorname{sign}(\gamma) A\right)
$$

which actually are all equivalent to the form originally proposed in Refs. [18,22], where only vertical compression and thus positive $\gamma$ was considered. ${ }^{5}$ The integrated form of Eq. (27) (using the exponential technique for ODE's) is calculated as:

$$
A=A^{\max }-\left(A^{\max }-A_{0}\right) \exp \left(-\beta_{A}|\gamma|\right) .
$$

This form of the evolution equation represents the fact that anisotropy starts at $A_{0}$ and then approaches exponentially fast to its maximum for large $\gamma$. For isotropic initial configuration, $A_{0}=0$, the growth is linear for small deformations $\gamma$, where the parameter $\beta_{A}$ determines how fast the anisotropy changes and thus also how fast saturation is approached. Typical values for these parameters - from DEM simulations - are $\beta_{A} \approx 80$, observed at small shear strain, and $A^{\max } \approx 0.4 B$, observed at large strain.

\subsubsection{Interpretation of the model}

When $A_{0}=0$ the stress evolution equations (23) and (24) take the simple linear elastic form as shown in Eqs. (25) and (26). The hydrostatic stress grows linearly with isotropic strain and the shear stress is proportional to the shear strain. However, the underlying physics changes since anisotropy can develop to $A \neq 0$.

The presence of the $A$ term creates a cross-link between the two types of deformations, shear and isotropic. This is best visualized considering a pure shear deformation, i.e., $\varepsilon^{v}=0$. In this case, for $A \neq 0$, the hydrostatic stress will start developing although the deformation is isochoric. This can be viewed differently as well: The build-up of stresses during isochoric shear deformation is due to the anisotropy modulus in Eq. (23). In a closed boundary system the material wants to expand (compact), dependent on its initial overconsolidated (underconsolidated) state. But since it is confined by the boundaries, the isochoric shear deformation will cause negative (positive) stresses in the anisotropic system. For example, in an overconsolidated situation, the explanation for this phenomenon is the interlocking of particles in the initial configuration. The system can not deform easily unless it is relaxed. The relaxation means that the system wants to create more voids and hence expand/dilate.

Similar considerations, in the isobaric situation, are discussed in more detail in Ref. [36] with respect to ratcheting under cyclic loading.

The second equation (24) predicts that under purely isotropic compressive strain, the shear stress will increase or decrease, dependent on the sign of the term involving $\varepsilon^{v}$. Correspondingly, compression and extension will lead to positive or negative changes of $\tau$, respectively. This result is plausible as it ensures symmetry in the system. If the stiffness is not isotropic, under compression, stress will develop differently in different directions. This will lead to principal stresses different from each other, hence shear stresses.

\footnotetext{
${ }^{4}$ Assume horizontal compression, which corresponds to $\gamma /|\gamma|<0$, that leads to an increase of horizontal stiffness and thus a positive $A^{\mathrm{max}}$. Correspondingly, compression in vertical direction leads to a negative $A^{\text {max }}-$ while tension in horizontal or vertical direction lead to negative and positive $A^{\mathrm{max}}$, respectively.

${ }^{5}$ The incremental anisotropy, here, is only defined for bi-axial deformation modes, i.e., for $\phi_{\varepsilon}=0$ (horizontal extension and vertical compression deviatoric strain) or $\phi_{\varepsilon}=\pi / 2$ (horizontal compression and vertical extension). A more general representation will be specified elsewhere.
} 


\subsubsection{Energy, work, stability}

In the linear elastic regime, the model can be integrated and leads to the free energy increment:

$$
\delta F=B\left(\delta \varepsilon^{v}\right)^{2}+G(\delta \gamma)^{2}+A \delta \varepsilon^{v} \delta \gamma,
$$

which allows to recover Eqs. (23) and (24) by differentiation of $F$ with respect to isotropic and deviatoric strain, respectively. This shows how to introduce our model into the framework of (linear elastic) GSH [33,34], i.e., by adding the additional anisotropy term to the elastic energy in Eq. (1) in Ref. [33].

Another important issue is the instability of the particulate system. This can be analyzed numerically using the system of equations introduced earlier. The sufficient but not necessary stability condition for materials is [37]:

$$
\delta^{2} W=\delta \boldsymbol{\sigma}: \delta \varepsilon \geq 0,
$$

which is equivalent in this case to:

$$
\delta \varepsilon: \mathbf{C}: \delta \varepsilon \geq 0
$$

For Eq. (30) to have a solution, $\mathbf{C}$ must be positive-definite. This implies that at the onset of instability, $\mathbf{C}$ will not be positive-definite anymore. In this case it can be checked by considering the matrix form given in Equation (19). For the $\mathbf{C}$ matrix to be singular its determinant must vanish, i.e., $\operatorname{det} \mathbf{C}=0$. Evaluating this analytically gives:

$$
\begin{gathered}
C_{11} C_{22}-C_{12}^{2}=0 \\
\Rightarrow(2 B-A-\lambda)(2 B+A-\lambda)-\lambda^{2}=0 \\
\Rightarrow(B+G-A)(B+G+A)-(B-G)^{2}=4 B G-A^{2}=0 .
\end{gathered}
$$

Using Eq. (31), the value of $A$ that will cause the system to become unstable can be found. For a typical case where $G=B / 2$, the system will become unstable only when $A$ reaches a value of $\sqrt{2} B$. Note, that this is not (yet) consistent with typically observed values of $A^{\max } \approx 0.4 B$.

\subsection{Non-linear stress evolution}

It is observed from DEM simulations, in bi-axial deformation mode 3, see table 1 below, that the response of the system to deformation is not always linear. As the amount of strain gets larger, the stress increments decrease until a level where the stress saturates, which is referred to as the critical state [38]. In Refs. [18,22], the evolution equation that leads to saturation under vertical compression and horizontally prescribed stress, is expressed as:

$$
\frac{\partial s_{d}}{\partial \gamma}=\beta_{s} \operatorname{sign}(\gamma)\left(s_{d}^{\max }-s_{d}\right)
$$

where $s_{d}$ is the stress deviator ratio, given by:

$$
s_{d}=\frac{\sigma_{11}-\sigma_{22}}{\left(\sigma_{11}+\sigma_{22}\right)}=\frac{\tau}{\sigma^{h}},
$$

and $s_{d}^{\max }=-\left|s_{d}^{\max }\right| \gamma /|\gamma|=-s_{d}^{m} \gamma /|\gamma|=-s_{d}^{m} \operatorname{sign}(\gamma)$, with (positive) maximum deviatoric stress ratio $s_{d}^{m}$. There are two reasons to use $s_{d}$ (instead of $\tau$ ). First, it is very similar to the MohrCoulomb plastic yield criterion, $\mu \equiv s_{d}^{m}$, and second, as empirical observation, $s_{d}^{m}$ does not much depend on the confining stress $[18,22,38]$ - which suggests that it is a material parameter (or at least a zero-order starting point).

The derivative of $s_{d}$ with respect to $\gamma$ reads

$$
\frac{\partial s_{d}}{\partial \gamma}=\frac{\partial}{\partial \gamma}\left(\frac{\tau}{\sigma^{h}}\right)=\frac{1}{\sigma^{h}}\left(\frac{\partial \tau}{\partial \gamma}-\frac{\tau}{\sigma^{h}} \frac{\partial \sigma^{h}}{\partial \gamma}\right),
$$


which, when inserting Eq. (32) and resolving with respect to $\partial \tau / \partial \gamma$, leads to

$$
\frac{\partial \tau}{\partial \gamma}=\sigma^{h} \beta_{s} \operatorname{sign}(\gamma)\left(s_{d}^{\max }-s_{d}\right)+\frac{\tau}{\sigma^{h}} \frac{\partial \sigma^{h}}{\partial \gamma}=2 G\left(1-s_{d} / s_{d}^{\max }\right)+s_{d} A
$$

The second right hand side term is obtained when the second term of Eq. (23) is inserted. The first right hand side term is based on the identification of Eq. (35) with Eq. (24), for very small strain. For $A_{0}=\tau_{0}=0$ we identify $2 G_{0}=-\sigma_{0} \beta_{s}^{0} s_{d}^{m}$ or $\beta_{s}^{0}=-2 G_{0} /\left(\sigma_{0} s_{d}^{m}\right)$, where the dependence on the initial isotropic stress, $\sigma_{0}$, is not fully consistent with observations from DEM simulations, see the discussion around Eq. (22) in Ref. [18]. It requires further study to understand the hidden explicit dependence of $G_{0}$ or $s_{d}^{m}$ on the hydrostatic stress.

On the other hand, for very large strain, one has $s_{d} \rightarrow s_{d}^{\max }$, i.e., the first term in Eq. (35) vanishes so that

$$
\frac{\partial \tau}{\partial \gamma}=s_{d}^{\max } \frac{\partial \sigma^{h}}{\partial \gamma}=s_{d}^{\max } A^{\max }
$$

remains, while $\partial s_{d} / \partial \gamma \rightarrow 0$.

Relying on the above observations, and requiring a "critical state flow" regime where all derivatives with respect to $\delta \gamma$ vanish after large enough deviatoric strain (with conserved volume $\delta \varepsilon^{v}=0$ ), a phenomenological extension of the linear model described in Eqs. (23) and (24) is proposed (in two versions) below.

\subsection{Improved non-linear Model}

In order for the deviator stress to vanish at large strain, the simplest possibility is to disregard the last term in Eq. (35), so that Eq. (24) is modified by a factor that vanishes when $s_{d}$ reaches its $\operatorname{maximum} s_{d}^{\max }$ :

$$
\delta \tau=A \delta \varepsilon^{v}+2 G\left(1-\frac{s_{d}}{s_{d}^{\max }}\right) \delta \gamma=A \delta \varepsilon^{v}+2 G S \delta \gamma
$$

using the abbreviation $S=\left(1-s_{d} / s_{d}^{\max }\right)$, i.e., the stress-isotropy, which varies between zero (maximal anisotropy in strain direction) and unity (isotropic), up to values of two (maximal anisotropy rotated by 90 degrees from the strain direction). With this variable $S$, another parameter is introduced in the model, namely the macroscopic coefficient of friction, $s_{d}^{m}$. The results of the DEM simulations [18], for example, show values $s_{d}^{m} \approx 0.4$, dependent strongly in the contact coefficient of friction of the material, but only weakly dependent on the confining stress $[18,22,38]$.

\subsubsection{Improved self-consistent non-linear Model}

Inserting the new deviatoric modulus, $\partial \tau / \partial \gamma=2 G S$, from Eq. (36) into Eq. (34), shows the inconsistency of the model in Eq. (36), i.e.,

$$
\frac{\partial s_{d}}{\partial \gamma}=\frac{1}{\sigma^{h}}\left(2 G\left(1-\frac{s_{d}}{s_{d}^{\max }}\right)-\frac{\tau}{\sigma^{h}} \frac{\partial \sigma^{h}}{\partial \gamma}\right)=\frac{1}{\sigma^{h}}\left(2 G S-s_{d} A\right),
$$

does not vanish for large deviatoric strain - in conflict with the critical state flow assumption. The consistency of the model can be restored by also correcting the second term in Eq. (23), analogously to the deviatoric modulus, by the factor $S$, so that the complete constitutive model reads:

$$
\begin{gathered}
\delta \sigma^{h}=2 B \delta \varepsilon^{v}+A S \delta \gamma \\
\delta \tau=A \delta \varepsilon^{v}+2 G S \delta \gamma
\end{gathered}
$$

and

$$
\delta A=\beta_{A} \operatorname{sign}(\gamma)\left(A^{\max }-A\right) \delta \gamma
$$


with three field quantities $\sigma^{h}, \tau$, and $A$ and five material model parameters: the bulk and shear moduli $B, G$, the macroscopic coefficient of friction, $s_{d}^{m}$, as well as the evolution rate of anisotropy from an isotropic original state, $\beta_{A}$, and the maximal anisotropy $A^{m}$. Furthermore, the initial conditions $\sigma_{0}, S_{0}$ and $A_{0}$ are required, where the abbreviation $S=\left(1-s_{d} / s_{d}^{\max }\right)$ was used.

How to measure the material model parameters will be discussed below and in future studies.

\subsubsection{Check of consistency}

Inserting the deviatoric modulus, $\partial \tau / \partial \gamma=2 G S$, from Eq. (39), and the anisotropic modulus $\partial \sigma^{h} / \partial \gamma=A S$, from Eq. (38), into Eq. (34), shows the consistency of the model, i.e.,

$$
\frac{\partial s_{d}}{\partial \gamma}=\frac{1}{\sigma^{h}}\left(2 G\left(1-\frac{s_{d}}{s_{d}^{\max }}\right)-\frac{\tau}{\sigma^{h}} \frac{\partial \sigma^{h}}{\partial \gamma}\right)=\frac{1}{\sigma^{h}}\left(2 G-s_{d} A\right) S,
$$

does vanish for large deviatoric strain.

Combining Eq. (32) with (41) leads to

$$
-\beta_{s} \sigma^{h} s_{d}^{m}=\left(2 G-s_{d} A\right),
$$

which, starting from an isotropic sample, for very small strain, with $A_{0}=\tau_{0}=0$, again leads to $2 G_{0}=-\sigma_{0}^{h} \beta_{s} s_{d}^{m}$ or $\beta_{s}=-2 G_{0} /\left(\sigma_{0}^{h} s_{d}^{m}\right)$, as before. For very large strain, one has $s_{d} \rightarrow s_{d}^{\max }$, i.e., $S \rightarrow 0$, so that, according to Eq. (41), one has $\partial s_{d} / \partial \gamma \rightarrow 0$. Furthermore, since $A \rightarrow A^{\max }$, and isobaric pure shear, one also might require to have:

$$
-\beta_{s} \sigma_{0}^{h} s_{d}^{m}=2 G_{0}=\left(2 G^{\max }-s_{d}^{\max } A^{\max }\right),
$$

which (unfortunately) is inconsistent again, unless $2 G \neq 2 G_{0}$, i.e., the modulus $G$ requires an evolution equation by itself, that must fulfill the consistency relation: $G^{\max }=G_{0}+(1 / 2) s_{d}^{\max } A^{\max }$. Since typically $(1 / 2) s_{d}^{\max } A^{\max }<G_{0}$, we assume $G \approx G_{0}=$ const. in the following.

\subsection{Alternative anisotropy evolution model}

Whether Eq. (40) has to be modified in a way similar to the second terms in Eqs. (38) and (39), which would lead to:

$$
\delta A=\beta_{A} \operatorname{sign}(\gamma)\left(A^{\max }-A\right) S \delta \gamma,
$$

will be discussed elsewhere. Since it is not necessary to achieve $\partial A / \partial \gamma \rightarrow 0$ for large deviatoric strains, we disregard this option and continue using Eq. (40) instead. Note that this keeps the evolution of $A$ most independent from the evolution of $s_{d}$.

\subsection{Energy, work, stability for the non-linear model}

Again, examining the stability condition by considering the matrix form given in Eq. (19), which actually is equivalent to the split hydrostatic/deviatoric form:

$$
\left[\begin{array}{c}
\delta \sigma^{h} \\
\delta \tau
\end{array}\right]=\left[\begin{array}{cc}
2 B & A S \\
A & 2 G S
\end{array}\right] \cdot\left[\begin{array}{c}
\delta \varepsilon^{h} \\
\delta \gamma
\end{array}\right]
$$

leads to the condition $\left(4 B G-A^{2}\right) S=0$, which can be fulfilled by either having the term in brackets vanish, or by $S \rightarrow 0$, as it happens for large shear strain anyway. 


\section{Results}

In this section, a series of tests will be performed, using the proposed constitutive model in different variants. The goal is to demonstrate the behavior of the model for different modes of deformation, as shown in Table 1.

Mode 0 is purely isotropic, while mode 2 is purely deviatoric, i.e., volume conserving, which both take especially simple forms. Modes 1 and 3 are mixed modes, which are often applied experimentally. The uni-axial test is the superposition of an isotropic and a deviatoric test, whereas the bi-axial test involves mixed stress- and strain-control instead of completely prescribed strains. In our examples, the strain component $\varepsilon_{22}$ is prescribed together with the horizontal stress component, $\sigma_{11}$, which makes, e.g., the horizontal strain component, $\varepsilon_{11}$, and the vertical stress, $\sigma_{22}$, unknown variables. Many further possible deformation modes, like isobaric mode 4, where the isotropic stress is prescribed constant [36], are not discussed here.

\begin{tabular}{llccccl} 
& Mode & 1-dir. & 2-dir. & $\varepsilon^{v}$ & $\gamma$ & Description \\
\hline 0 & (isotropic) & $\varepsilon_{0}$ & $\varepsilon_{0}$ & $\varepsilon_{0}$ & 0 & $\varepsilon_{0}>0$ dilatation, $\varepsilon_{0}<0$ compression \\
\hline \multirow{2}{*}{1} & (uniaxial) & $\varepsilon_{1}$ & 0 & $\frac{\varepsilon_{1}}{2}$ & $\frac{\varepsilon_{1}}{2}$ & horizontal, $\varepsilon_{1}>0$ tension, $\varepsilon_{1}<0$ compression \\
\cline { 2 - 7 } & 0 & $\varepsilon_{1}$ & $\frac{\varepsilon_{1}}{2}$ & $-\frac{\varepsilon_{1}}{2}$ & vertical, $\varepsilon_{1}>0$ tension, $\varepsilon_{1}<0$ compression \\
\hline 2 & (deviatoric) & $\varepsilon_{2}$ & $-\varepsilon_{2}$ & 0 & $\varepsilon_{2}$ & pure shear, $\varepsilon_{2}>0$ positive, $\varepsilon_{2}<0$ negative \\
\hline 3 & (bi-axial) & $\sigma_{11}=\sigma_{0}$ & $\varepsilon_{22}$ & $?$ & $?$ & horizontal stress- and vertical strain-control \\
\hline
\end{tabular}

Table 1: Summary of various deformation modes that can be imposed on the system. The term 1-dir. is the abbreviation for $x$ - or 1 -direction. The symbols $\varepsilon_{\alpha}$ with $\alpha=0,1,2,3$ indicate the magnitude of strain of the respective mode and should not be confused with eigen-values or a-like. In the mode 3 bi-axial test one has $\sigma_{11}=\sigma_{0}=$ const. and $\varepsilon_{22}$ is a (smooth) prescribed function of time.

In the following, different cases of the constitutive model will be discussed, starting from isotropy, $A=0$, and constant anisotropy $A=A_{0}$, before different variants of the non-linear, anisotropic model are studied.

\subsection{Isotropy $A=0$}

The isotropic special case is realized by setting $A=A^{\max }=\beta_{A}=0$, which de-couples the evolution equations for the isotropic and deviatoric stresses and allows for a straightforward integration given the constants $B, G$, and $s_{d}^{\max }$, with initial conditions $\sigma_{0}=\sigma_{0}^{h}$ and $S_{0}=\tau_{0} / \sigma_{0}$.

\subsection{Constant Anisotropy $A=A_{0}$}

In this subsection the cases where $A$ is constant are discussed, which implies that the anisotropy of the system is not changing with the deformation. Isotropy is a special case belonging to this class. For the sake of simplicity, in the constitutive model Eqs. (38) and (39), we set the stress isotropy to $S=1$; variations in $S$ will be discussed in the next subsection.

Note that this subsection is relevant for very small deformations of systems with initial values of $B_{0}, G_{0}, A_{0}$ and $S_{0}$. With other words, this subsection describes the stress-strain response of the model for infinitesimal deformations from an arbitrary initial configuration. 


\subsubsection{Linear model, mode 0 (isotropic) and constant $A=A_{0}$}

The first example shows the effects of pure isotropic deformation (mode 0) on the shear stress. The prescribed deformation consists of $\varepsilon_{11}=\varepsilon_{22}=\varepsilon_{0}$ and volume change $2 \varepsilon^{v}=2 \varepsilon_{0}<0$, i.e., compression. The stresses that develop during the deformation, with constant $S=1$, are shown in Figure 2.
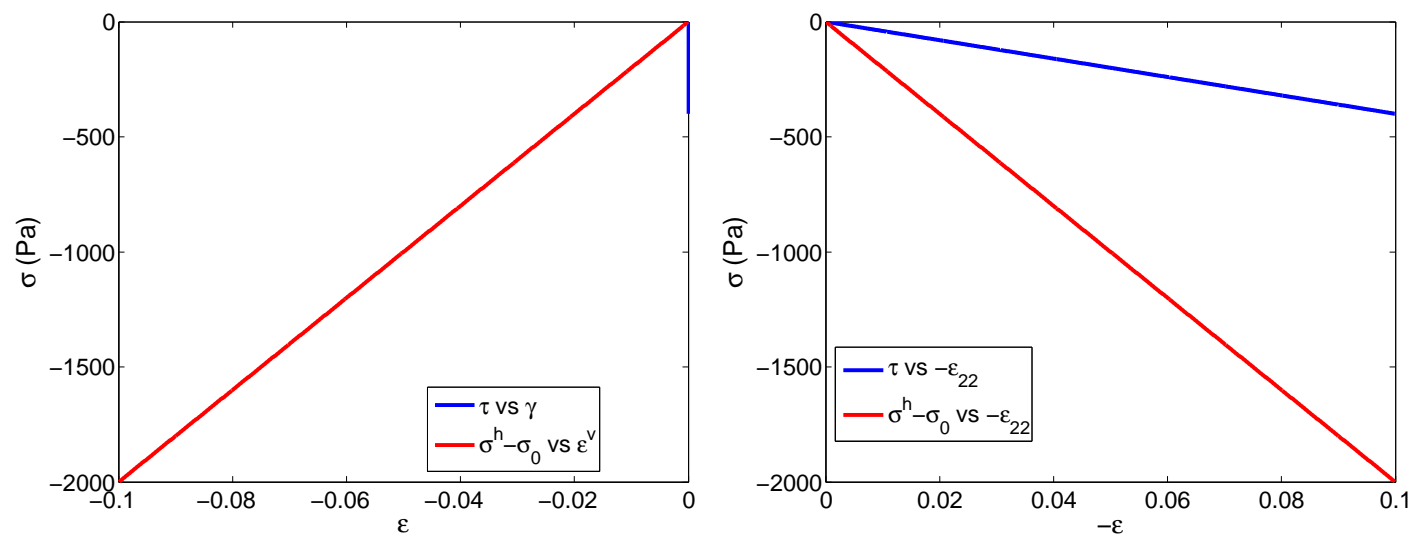

Figure 2: The development of shear and hydrostatic stresses under isotropic compression (mode 0 ) for fixed $A=A_{0}=2 B / 5$, with parameters $\sigma_{0}=-100 \mathrm{~Pa}, B=10000 \mathrm{~Pa}$, and $G=B / 2$.

The compression test leads to increasing negative isotropic stress. Furthermore, even though there is no shear deformation in the system, $\gamma=0$, the shear stress $\tau$ changes for $A \neq 0$. For this example system, recall that positive $A$ corresponds to a larger horizontal than vertical stiffness. Therefore, the (deviatoric) shear stress-response, $\tau$, must be negative, i.e., the horizontal stress (negative) increases stronger than the vertical stress (negative).

In the case of negative $A<0$, the isotropic stress remains unchanged, but the shear stress, $\tau$, changes sign, i.e., the vertical stress (negative) increases stronger than the horizontal stress (negative) and thus $\tau>0$.

When the system is expanded, i.e., $\varepsilon_{0}>0$, the stresses will develop in the opposite (positive) direction - the isotropic stress will increase, while (for $A>0$ ) the deviatoric stress increases to positive values too, i.e., the horizontal stress increases stronger than the vertical stress, leading to positive shear stress $\tau$.

Best Practice Tip 1: Applying a purely isotropic deformation (mode 0) to a sample allows to measure the modulus $2 B$ and the anisotropy of the sample, $A$, from the initial slopes of $\sigma^{h}-\sigma_{0}$ and $\tau$, respectively, plotted against $\varepsilon^{v}$. (In the linear model with constant $A$ and $S$, the slopes do not change with increasing strain, whereas in the general case below, they do.)

\subsubsection{Linear model, mode 2 (deviatoric) and constant $A=A_{0}$ with varying $S$}

The next example shows a case where the prescribed deformation is pure shear (mode 2), with $\varepsilon_{11}=-\varepsilon_{22}=\varepsilon_{2}=\gamma>0$, i.e., for compression in vertical and expansion in horizontal direction. In Figure 3 the development of the deviatoric stress $(\equiv \tau)$ and the hydrostatic stress are shown.

Even though there is no isotropic strain involved, the model leads to an increase of the hydrostatic stress, i.e., a reduction of the compression. This is due to $A>0$, so that the horizontal stress increases faster (tensile) than the vertical stress decreases (compressive). If the volume would not be prescribed as constant, the constitutive model would lead to compaction, i.e., decrease of volume of the anisotropic system due to shear deformation. 


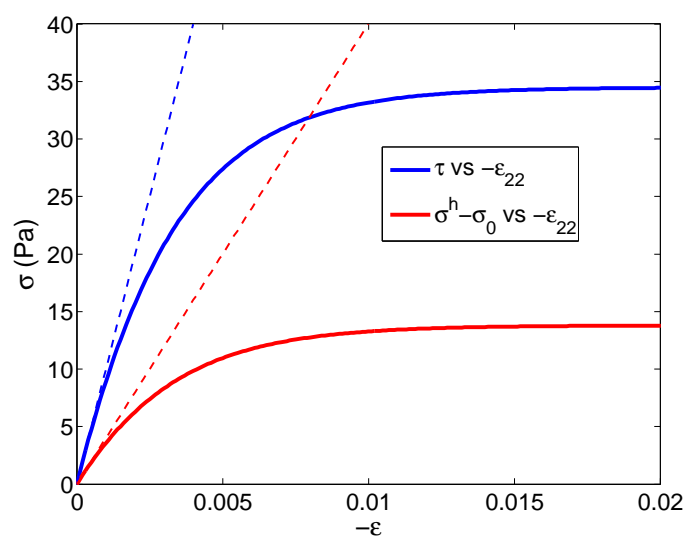

Figure 3: The development of shear and hydrostatic stresses under pure shear deformation, mode 2 , for fixed $A=A_{0}=2 B / 5$, with $\sigma_{0}=-100 \mathrm{~Pa}, B=10000 \mathrm{~Pa}, G=B / 2, s_{d}^{\max }=0.4$, and $S_{0}=1$. The dashed lines indicate the initial slopes $2 G$ and $A$ and correspond to constant $S=S_{0}=1$.

Inversion of the sign of $A$ does not affect the shear stress, but leads to a system with stiffer response in vertical direction and, correspondingly, to a sign reversal of the hydrostatic stress change and therefore dilatancy instead of compaction. Reversal of the shear direction, but keeping $A>0$, just inverts all signs for both strain and stress changes.

Best Practice Tip 2: Applying a purely deviatoric deformation (mode 2) to a sample allows to measure the modulus $2 G S$ and the anisotropy, AS from the initial slopes for small strain. Note that, as shown below, the measured moduli depend also on the initial state of $S$-only for isotropic initial stress, $S=1$, the parameters $2 G$ and $A$ are directly available.

\subsubsection{Comparison of mode 0 and mode 2 for constant $A=A_{0}$}

Comparison of the two cases, mode 0 and mode 2, reveals an important characteristics of the model. There exists a coupling symmetry or, in other words, the cross-link between shear (deviatoric) and isotropic strains works in both directions: shear strain causes a change in hydrostatic stress and isotropic strain causes a change in shear (deviatoric) stress - if anisotropy is present.

\subsubsection{Linear model, Mode 1 for constant $A=A_{0}\left(\right.$ and $\left.S=S_{0}=1\right)$}

Applying uni-axial deformation (mode 1) with strain $\varepsilon_{1}$, in horizontal or vertical direction, leads to:

$$
\delta \sigma^{h}=B \delta \varepsilon_{1} \pm(A / 2) \delta \varepsilon_{1}=(2 B \pm A) \delta \varepsilon_{1}^{v}=( \pm 2 B+A) \delta \gamma_{1},
$$

and

$$
\delta \tau=(A / 2) \delta \varepsilon_{1} \pm G \delta \varepsilon_{1}=(A \pm 2 G) \delta \varepsilon_{1}^{v}=( \pm A+2 G) \delta \gamma_{1},
$$

respectively, where the positive/negative, \pm , corresponds to horizontal/vertical deformation with corresponding shear strain $\delta \gamma_{1}>0$ or $\delta \gamma_{1}<0$. Inserting our choice of parameters, $A=2 B / 5$ and $G=B / 2$, yields: $\delta \sigma^{h}=(1 \pm 1 / 5) B \delta \varepsilon_{1}$, and $\delta \tau=(1 / 5 \pm 1 / 2) B \delta \varepsilon_{1}$.

Note that for the simplest uni-axial (mode 1) deformation experiments, typically only the stress in one direction (deformation direction) can be measured. Therefore, by addition or subtraction of Eqs. (44) and (45), the relations

$$
\delta \sigma_{11}=(B+G+A) \delta \varepsilon_{11},
$$


and

$$
\delta \sigma_{22}=(B+G-A) \delta \varepsilon_{22},
$$

can be used - if the horizontal or vertical stresses are measured for the respective horizontal or vertical deformation direction.

For these cases, when the deformation direction is reversed, $\delta \varepsilon_{1} \rightarrow-\delta \varepsilon_{1}$, one obtains the same moduli (due to sign inversion of the stress response). The model with constant $A$ thus does not show non-linearity under reversal of deformation direction. On the other hand, when the anisotropy direction is reversed, $A \rightarrow-A$, the apparent moduli for horizontal or vertical deformation are exchanged in Eqs. (46) and (47).

If the experiment also allows to measure the stress perpendicular to the uni-axial deformation direction, one has the relations

$$
\delta \sigma_{22}=(B-G) \delta \varepsilon_{11},
$$

and

$$
\delta \sigma_{11}=(B-G) \delta \varepsilon_{22},
$$

i.e., three apparent moduli can be measured to determine the three unknowns $B, G$, and $A$. The fourth unknown $S_{0}$ will be introduced in the next sub-subsection. Note that in practice uni-axial experiments are usually not flexible enough to provide all four measurement results. From DEM simulations, however, one can easily obtain the three moduli, given the system is initially isotropic in stress, $S_{0}=1$.

Best Practice Tip 3.1: For isotropic stress $S=S_{0}=1$, the uni-axial deformation mode does not provide direct access to the constitutive model parameters - however, various combinations can be obtained for different tests, dependent on the direction of deformation and initial anisotropy. In particular, combinations like $B \pm G$ and $A$ can be extracted.
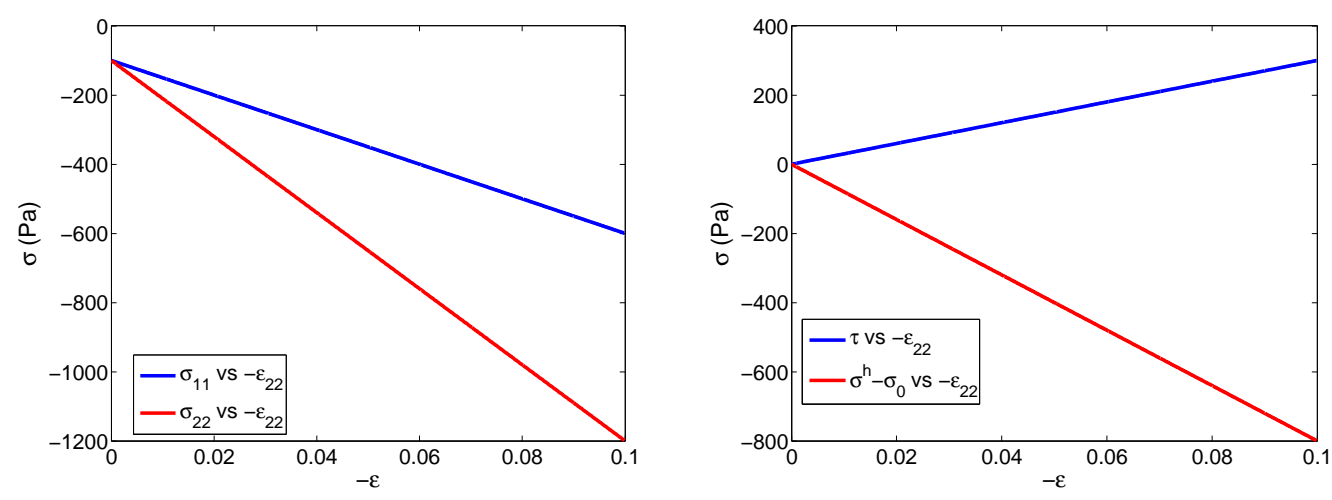

Figure 4: (Left) The horizontal and vertical stresses under vertical uni-axial deformation, mode 1 , plotted as function of the negative vertical strain (such that compression appears positive). (Right) shear and hydrostatic stresses from the same data, also plotted against (negative) vertical strain. The data are for fixed $A$, with $\sigma_{0}=-100 \mathrm{~Pa}, B=10000 \mathrm{~Pa}, G=B / 2, A=A_{0}=2 B / 5$ and $S=S_{0}=1$. The apparent moduli, $i$.e., the slopes of the curves in the left panel are $-(B+G-A)=$ $-11000 \mathrm{~Pa}$ for the vertical stress, see Eq. $(47)$, and $-(B-G)=-5000 \mathrm{~Pa}$ for the horizontal stress, see Eq. (49). The slopes in the right panel are $-8000 \mathrm{~Pa}$ and $+3000 \mathrm{~Pa}$, as can be read off from Eqs. (44) and (45), for the hydrostatic and the deviatoric stress, respectively.

Examples of the linear model for uni-axial compression, mode 1, and how to obtain the apparent moduli from the previous set of equations, are given in Figs. 4 and 5. In the following subsection, the same system will be examined with initial anisotropy $S<1$. 

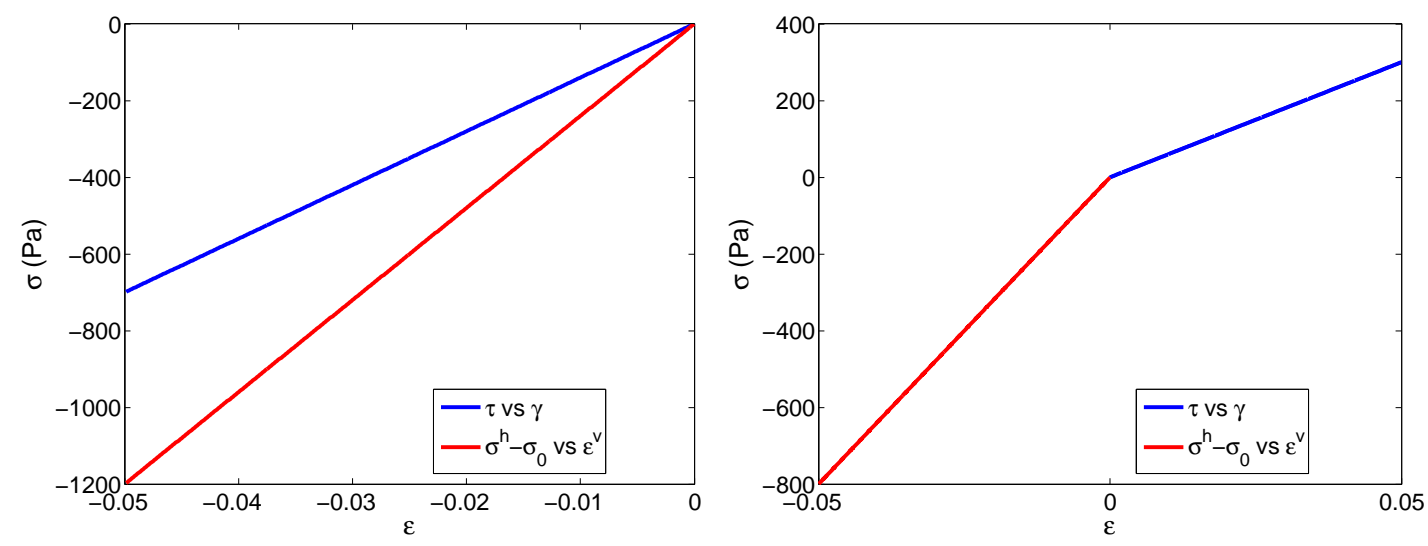

Figure 5: The development of shear and hydrostatic stresses under horizontal (Left) and vertical (Right) uni-axial deformation, mode 1 , for fixed $A$, with $\sigma_{0}=-100 \mathrm{~Pa}, B=10000 \mathrm{~Pa}, G=B / 2$, $A=A_{0}=2 B / 5$ and $S_{0}=1$. In the right panel, for vertical uni-axial compression, in contrast to Fig. 4, the hydrostatic and deviatoric stresses are plotted against the isotropic and deviatoric strains, respectively. In the right panel, the corresponding slopes $2 B-A=16000 \mathrm{~Pa}$ and $2 G-A=$ $6000 \mathrm{~Pa}$ can also be read off from Eqs. (44) (2nd term) and (45) (third term). In the left panel, for horizontal uni-axial compression, the slopes are $2 B+A=24000 \mathrm{~Pa}$ and $2 G+A=14000 \mathrm{~Pa}$.

\subsubsection{Linear model, Mode 1 for constant $A=A_{0}$ (and $S=S_{0} \neq 1$ )}

Applying uni-axial deformation (mode 1 ), with strain $\delta \varepsilon_{1}$ to the (deviatorically) pre-stressed system (with $S_{0} \neq 1$ ), in horizontal or vertical direction, leads to

$$
\delta \sigma^{h}=B \delta \varepsilon_{1} \pm(A / 2) S_{0} \delta \varepsilon_{1},
$$

and

$$
\delta \tau=(A / 2) \delta \varepsilon_{1} \pm G S_{0} \delta \varepsilon_{1},
$$

respectively. This leads to the modified relations for the simplest uni-axial test

$$
\delta \sigma_{11}=\left(B+G S_{0}+A / 2+A S_{0} / 2\right) \delta \varepsilon_{11},
$$

and

$$
\delta \sigma_{22}=\left(B+G S_{0}-A / 2-A S_{0} / 2\right) \delta \varepsilon_{22},
$$

which can be used if the horizontal and vertical stresses are measured for the respective horizontal and vertical deformation directions. If the experiment also allows to measure the stress perpendicular to the uni-axial deformation direction, one has the independent relations

$$
\delta \sigma_{22}=\left(B-G S_{0}-A / 2+A S_{0} / 2\right) \delta \varepsilon_{11},
$$

and

$$
\delta \sigma_{11}=\left(B-G S_{0}+A / 2-A S_{0} / 2\right) \delta \varepsilon_{22},
$$

i.e., four relations for the four unknowns $B, G, A$, and $S_{0}$.

Best Practice Tip 3.2: The uni-axial deformation mode does not provide direct access to the constitutive model parameters - however, various combinations can be obtained in different realizations, dependent on the direction of deformation and initial anisotropy. In particular combinations like $B \pm G S_{0}$ and $A\left(1 \pm S_{0}\right) / 2$ can be extracted, from the initial slopes. 


\subsubsection{Comment on direction reversal of uni-axial deformation}

Now a final remark about the non-linearity under direction reversal. Like above for $S=1$, for constant $A=A_{0}$ and constant $S=S_{0}<1$, there is linearity for reversal of deformation-direction. However, as will be discussed in the following subsection, for $A$ and $S$ variable, developping during the applied strain history, the non-linearity in the model shows up.

More specific, assume the special case, where the sample was compressed (uni-axially) in horizontal direction (long enough, $\gamma \gg 0$ ) such that the final, critical state with $A=A_{c}^{\max }=A^{m}>0$, and $s_{d, c}^{\max }=-s_{d}^{m}<0$ was reached. Measuring from this state, uni-axially the horizontal stiffness under further horizontal compression implies $A_{0}=A^{m}$ and $S_{0}=0$ so that Eq. (52) leads to the apparent modulus $C_{1111}=\left(2 B+A^{m}\right) / 2\left(=6 B / 5\right.$ for our parameters with $\left.A^{m}=2 B / 5\right)$.

On the other hand, after stop and measuring from the same state, uni-axially the horizontal stiffness under the reversed (opposite) horizontal deformation (i.e., tension) implies sign reversal of strain and (due to the definitions of $A_{t}^{\max }=-A^{m}$, see Eq. (27), and $s_{d, t}^{\max }=s_{d}^{m}$, see Eq. (32)) implies $A=A_{0}=-A^{m}$ and $S_{0}=2$ (at the instant of direction reversal), which leads to the apparent modulus $C_{1111}^{\text {rev }}=B+2 G-3 A^{m} / 2$ (=14B/10 for our parameters). In general, the modulus under reversal, $C_{1111}^{\text {rev }}$, is different from the apparent modulus $C_{1111}$ under further uni-axial compression, which allows to quantify the strength of non-linearity via $\Psi^{\mathrm{rev}}=C_{1111}^{\mathrm{rev}} / C_{1111}=\frac{2 B+4 G-3 A^{m}}{2 B+A^{m}}(=7 / 6$ for our parameters). For $\Psi^{\text {rev }}>1$ one has an increased stiffness under strain reversal whereas for $\Psi^{\text {rev }}<1$, the stiffness would decrease under strain reversal. Since the former is usually observed in experiments and simulations, we can define an upper limit for $|A| \leq A_{\Psi}:=G$, for which $\Psi^{\mathrm{rev}} \equiv 1$.

\subsubsection{Mode 3 for $A=A_{0}$ with varying $S$}

The last example for constant $A$ is the actual bi-axial model system, i.e., mode 3 with constant horizontal stress. In this case only $\varepsilon_{22}$ is prescribed as compressive (negative) and $\varepsilon_{11}$ is calculated using the constraint condition $\sigma_{11}=\sigma_{0}$.
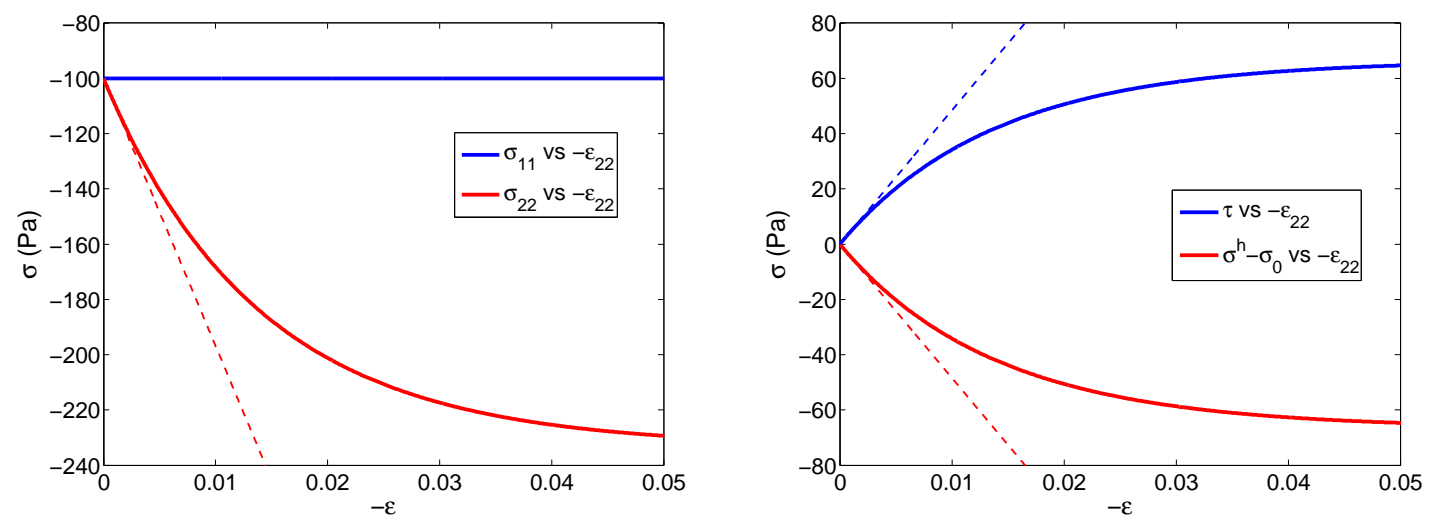

Figure 6: The development of stresses during the biaxial deformation (mode 3) of the system, plotted against the negative vertical strain. (Left) Horizontal and vertical stress, where the dashed line indicates the initial slope, $-C_{2222}$, and (Right) hydrostatic and deviatoric stress, where the dashed lines indicate the slopes $\pm C_{2222} / 2$, for a system with fixed $A=A_{0}=0.4 B, \sigma_{11}=\sigma_{0}=$ $-100 \mathrm{~Pa}, B=10000 \mathrm{~Pa}$, and $G=B / 2$.

Figure 6 shows the components of the stress tensor as well as the shear and hydrostatic stresses. The horizontal stress remains constant, as prescribed, and, for our choice of parameters, the shear stress increases with slope $C_{3}$ while the isotropic stress decreases with slope $-C_{3} .{ }^{6}$ The vertical

\footnotetext{
${ }^{6}$ The anti-symmetry in isotropic and deviatoric stress for mode 3 is due to the prescribed constant horizontal stress $\sigma_{11}=\sigma_{0}$, which implies $\delta \sigma_{11}=0$. The constant $C_{3}=C_{2222} / 2$ is derived below.
} 
stress $\sigma_{22}$ evolves according to the constitutive relations, i.e., it decreases to higher compressive stress magnitude (negative). Since $A$ is constant, the model leads to a linear increase of stress magnitude to values much larger than the confining stress.

Inserting the definitions into the constitutive model, at initial state with $S_{0}=1$, leads to:

$$
\begin{aligned}
& \delta \sigma^{h}=\delta \sigma_{22} / 2=2 B\left(\delta \varepsilon_{11}+\delta \varepsilon_{22}\right) / 2+A\left(\delta \varepsilon_{11}-\delta \varepsilon_{22}\right) / 2=2 B \delta \varepsilon^{v}+A \delta \gamma, \\
& \delta \tau=-\delta \sigma_{22} / 2=A\left(\delta \varepsilon_{11}+\delta \varepsilon_{22}\right) / 2+2 G\left(\delta \varepsilon_{11}-\delta \varepsilon_{22}\right) / 2=A \delta \varepsilon^{v}+2 G \delta \gamma,
\end{aligned}
$$

and setting $\delta A=0$, allows to solve for the unknowns

$$
\delta \varepsilon_{11}=-\frac{(B-G)}{(B+G+A)} \delta \varepsilon_{22}=-\nu_{P} \delta \varepsilon_{22},
$$

and

$$
\delta \sigma_{22}=\frac{\left[4 B G-A^{2}\right]}{2(B+G+A)} \delta \varepsilon_{22}=C_{2222} \delta \varepsilon_{22}
$$

where the first equation defines the Poisson ratio $\nu_{P}$ and the second equation directly provides the modulus $C_{2222}$ for the bi-axial compression mode. The Poisson ratio allows to relate the isotropic to the deviatoric strains via: $\varepsilon^{v}=-\frac{1-\nu_{P}}{1+\nu_{P}} \gamma$. Inserting our choice of parameters, $A=2 B / 5$ and $G=B / 2$, yields $\nu_{P}=5 / 19$, and $C_{2222}=92 B / 95$, which corresponds to the (negative) slope of $\sigma_{22}$ in Fig. 6.

Best Practice Tip 4.1: The bi-axial deformation mode does not provide direct access to the constitutive model parameters - however, other quantities like a Poisson ratio and one modulus can be obtained.

In Figure 7 both the evolution of the strains and the scaled stresses is shown, where the latter are defined as:

$$
s_{v}=\frac{\sigma_{11}+\sigma_{22}}{2 \sigma_{0}}-1, \quad \text { and } \quad s_{d}=\frac{\sigma_{11}-\sigma_{22}}{\left(\sigma_{11}+\sigma_{22}\right)} .
$$

In the left panel, we observe a continuous decrease of volume, $\delta \varepsilon_{v}<0$ (negative slope of the red curve), with initial slope $-\left(1-\nu_{P}\right) / 2=-7 / 19$, due to the relation $\varepsilon_{v}=\left(1-\nu_{P}\right) \varepsilon_{22} / 2$. The vertical strain is continuously decreasing while the horizontal strain increases - but more slowly which leads to a net decrease of volume (compaction). ${ }^{7}$ For the same reasons, the shear strain continuously increases according to $\gamma=-\left(1+\nu_{P}\right) \varepsilon_{22} / 2$, with initial slope $12 / 19$.

From the right panel, we observe that the isotropic stress ratio is continuously increasing (consistent with the isotropic compaction), according to $s_{v}=\sigma_{22} /\left(2 \sigma_{0}\right)=C_{3} \varepsilon_{22} / \sigma_{0}$, and the deviatoric stress ratio decreases as $s_{d}=-C_{3} \varepsilon_{22} / \sigma_{0}$. The deviatoric stress ratio, for large strains, approaches the value $s_{d}^{\max }=-0.4$, as prescribed, while the isotropic stress ratio approaches $s_{v}^{\max }=2 / 3$.

\subsection{Model with evolution of anisotropy $A$}

From discrete element simulations it is observed that $A$ evolves during the deformation according to the proposed function in Eq. (40) - before shear band formation, as long as the system is homogeneous. The model proposed in this study in Eqs. (38), (39), and (40), is therefore implemented for evolving $A$ - to be compared with DEM simulations in a future study. Here, results will be shown for the biaxial box deformation mode 3, i.e., for vertical compression and with fixed horizontal stress.

\footnotetext{
${ }^{7}$ Note that our positive $A=2 B / 5$ corresponds to a material that is stiffer in horizontal than in vertical direction. Inserting $A=-2 B / 5$ corresponds to a material that is stiffer in vertical directions. This leads to $\nu_{P}=5 / 11$, i.e., a considerably larger Poisson ratio than for positive $A$, while $A=0$ leads to a value in between. The volume change is given by $\varepsilon_{v}=\left(1-\nu_{P}\right) \varepsilon_{22} / 2$, so that $A=2 B / 5,0$, and $-2 B / 5$ lead to $\left(1-\nu_{P}\right)=0.736842,0.6666$, and 0.545454 , respectively. The higher the stiffness in compression direction, the smaller the volume change for given compression.
} 

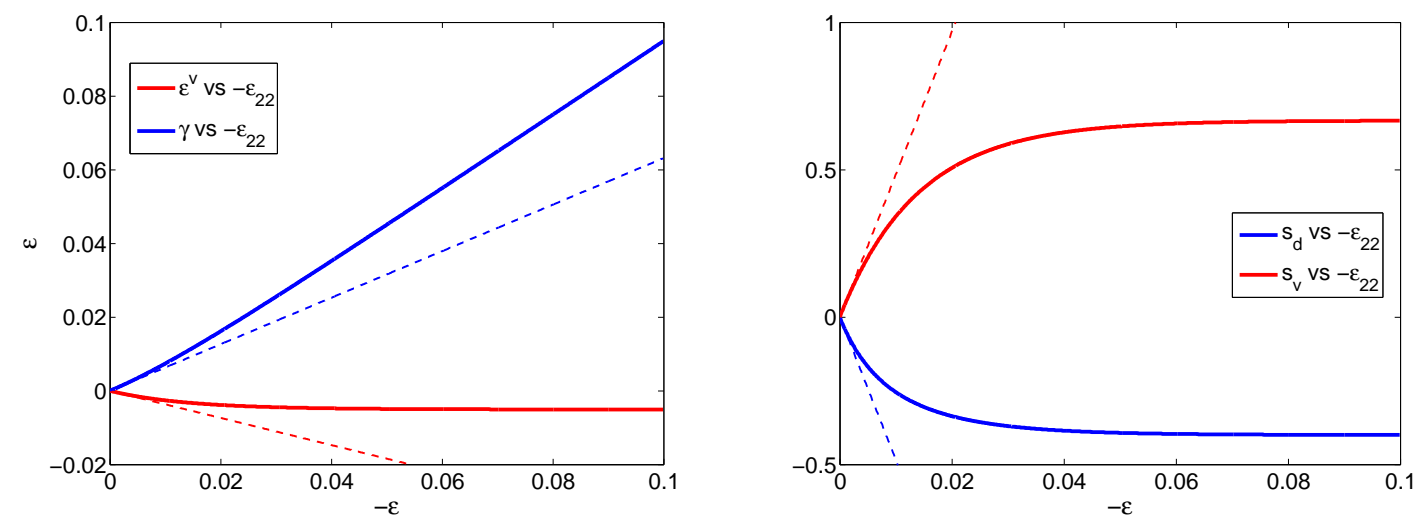

Figure 7: (Left) The development of isotropic and shear (deviatoric) strain during the biaxial deformation of the system, mode 3 , for fixed $A$, from the same data as in Fig. 6 , where the dashed lines indicate the initial slopes $-7 / 19$ and $12 / 19$. (Right) The development of the scaled stresses, $s_{v}$ and $s_{d}$, from the same data, where the slopes of the dashed lines are $\pm C_{3} / \sigma_{0}$.

\subsubsection{Biaxial mode 3 with vertical compression}

In the first example the maximal anisotropy is $A^{m}=2 B / 5$, like the constant $A$ before, however, the anisotropy $A$ is now free to change, starting from $A_{0}=0$, if not explicitly mentioned. The other parameters in the simulation are: $\sigma_{0}=-100 \mathrm{~Pa}, B=10000 \mathrm{~Pa}, G=B / 2, \beta_{A}=82$, and $s_{d}^{m}=0.4$.

The stresses and strains behave initially like for constant $A=A_{0}=0$ (data not shown). The behavior starts, as expected, being linear, isotropic and evolves towards being saturated, anisotropic. Note that this system, therefore, behaves differently than the system with constant $A \neq 0$, as discussed before in subsection 4.2.7.

In Fig. 8 (Left), the isotropic strain indicates the initial compression and then saturates at around $2 \%$ of vertical strain, while the deviatoric strain increases continuously with increasing slope, relative to the initial deformation, and constant slope later on. The continuously increasing shear strain is due to the horizontal extension and vertical compression, which both enter $\gamma$ positively. Note however, that the initial isotropy $A_{0}=0$ has as consequence that the volume change is smaller while the shear strain is larger - the initial slopes are $-1 / 3$ and $2 / 3$ for volumetric and deviatoric strain, respectively - as compared to the respective data with constant $A=A_{0}=0.4 B$. Also the saturation level of isotropic and deviatoric strain is different from the previous case.

In Fig. 8 (Right), the non-dimensional shear stress ratio, $s_{d}$, decreases and saturates at $s_{d}^{\max }=-0.4$, as prescribed. The isotropic stress ratio also saturates, at a positive value $s_{v}^{\max }$, which was not prescribed, but is due to the constant horizontal stress $\sigma_{11}=\sigma_{0}$, which together with the prescribed $s_{d}^{\max }$ does not allow the isotropic stress to change anymore beyond $s_{v}^{\max }=-\left(1 / s_{d}^{\max }+1\right)^{-1}=\frac{2}{3}$. Thus, the saturation values are independent of the initial value of $A$. The early evolution of the stress ratios, however, is influenced by the evolution of $A$, as can be seen by the difference between the dashed and solid lines. The negative anisotropy, $A<0$, starting from $A_{0}=0$ speeds up the evolution of the stress ratios, as compared to the positive $A$ situation.

The evolution of anisotropy $A$ is displayed in Fig. 9, and shows saturation at $A^{\max }=-2 B / 5$ at around $7 \%$ of strain for $\beta_{A}=82$. For larger $\beta_{A}$, saturation is much faster, while for smaller $\beta_{A}$, the change of $A$ is much slower. Negative $A$ means that the horizontal stiffness is smaller than the vertical stiffness, which is plausible due to the vertical compression and the horizontal extension.

The second-order mechanical work, as defined in Eq. (30), is shown in Fig. 9(right), and displays a rapid decay from large values down to zero in the anisotropy saturation regime. Interestingly, 

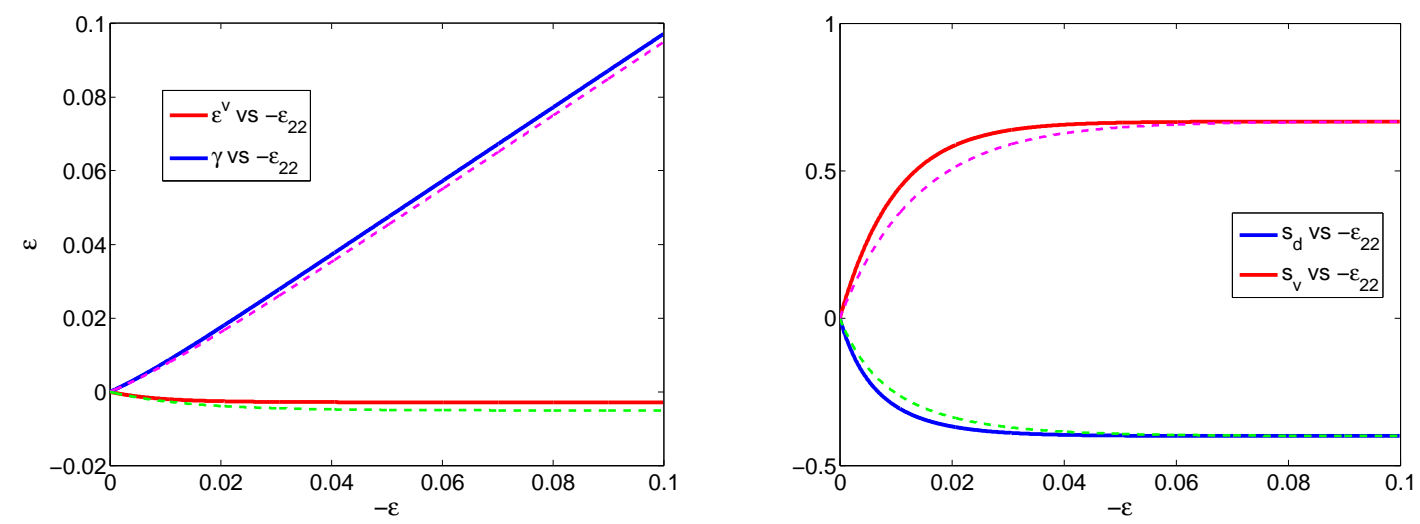

Figure 8: The development of shear and isotropic strains (Left) and of the non-dimensional stressratios, $s_{d}$ and $s_{v}$ (Right), during the biaxial deformation mode 3 of the system, with vertically prescribed compression and constant horizontal stress, $\sigma_{0}=-100 \mathrm{~Pa}$, for evolving $A$, using the standard parameters as given in the main text, together with the new parameters $\beta_{A}=82$, $A^{m}=2 B / 5$, and $s_{d}^{m}=0.4$. The solid lines are the data with variable $A$, whereas the dashed lines are the data for constant $A$ from Fig. 7.
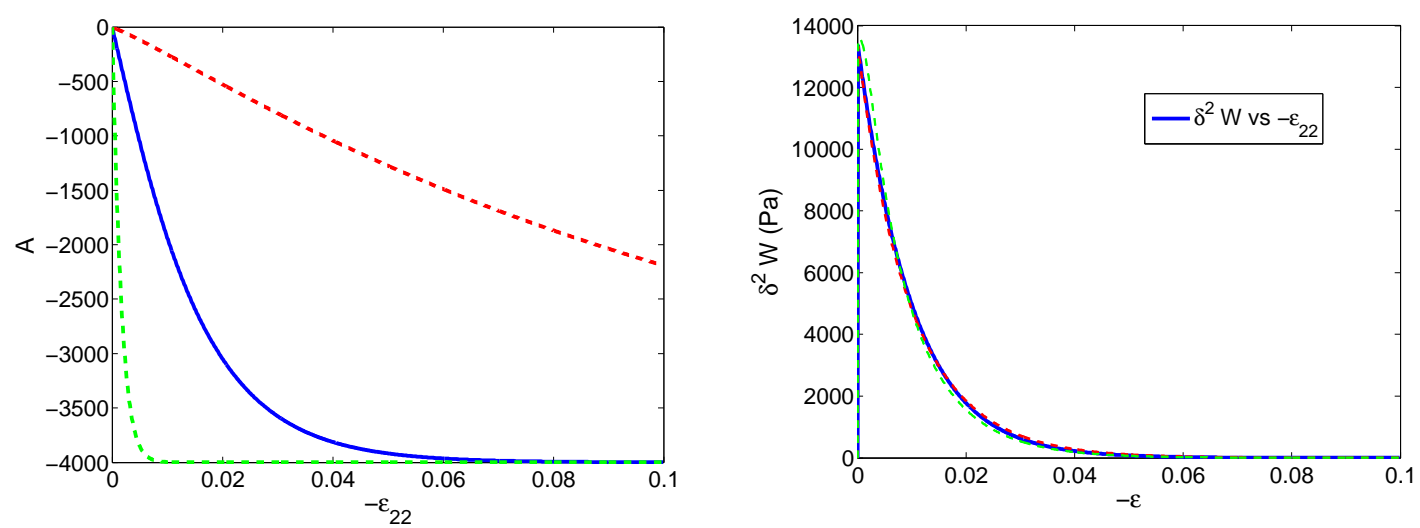

Figure 9: The evolution of anisotropy $A$ (Left) during mode 3 vertical compression, with constant horizontal confining stress $\sigma_{0}$, for three different $\beta_{A}=820,82$, and 8.2 (from bottom to top), and the development of second-order mechanical work (Right) from the same simulations.

the second order work is not much affected by $\beta_{A}$.

\subsubsection{Biaxial mode 3 with vertical extension}

The second example is identical to the first example, only reversing the vertical strain direction to extension, starting from $A_{0}=0$, with $A^{m}=2 B / 5, \sigma_{0}=-100 \mathrm{~Pa}, B=10000 \mathrm{~Pa}, G=B / 2$, $\beta_{A}=82$, and $s_{d}^{m}=0.4$.

For vertical extension, keeping the horizontal stress constant, the system response is initially the same as for compression - only with opposite signs for the strain, the anisotropy and the stress changes. This is due to the fact that we start from an isotropic system, $A_{0}=0$, i.e., like those shown in Fig. 10, all the quantities develop with the same slope from the starting point at zero strain. However, for larger strains the evolution of some of the quantities develops differently. The anisotropy is an exception here, since its evolution is symmetric with respect to the sign of strain - in the present simplified version of the model. The saturation values are equal in magnitude 
but opposite in sign, $A^{\max }= \pm 2 B / 5$, for extension (positive) and compression (negative) and the inital rate of change is only determined by $\beta_{A}$.
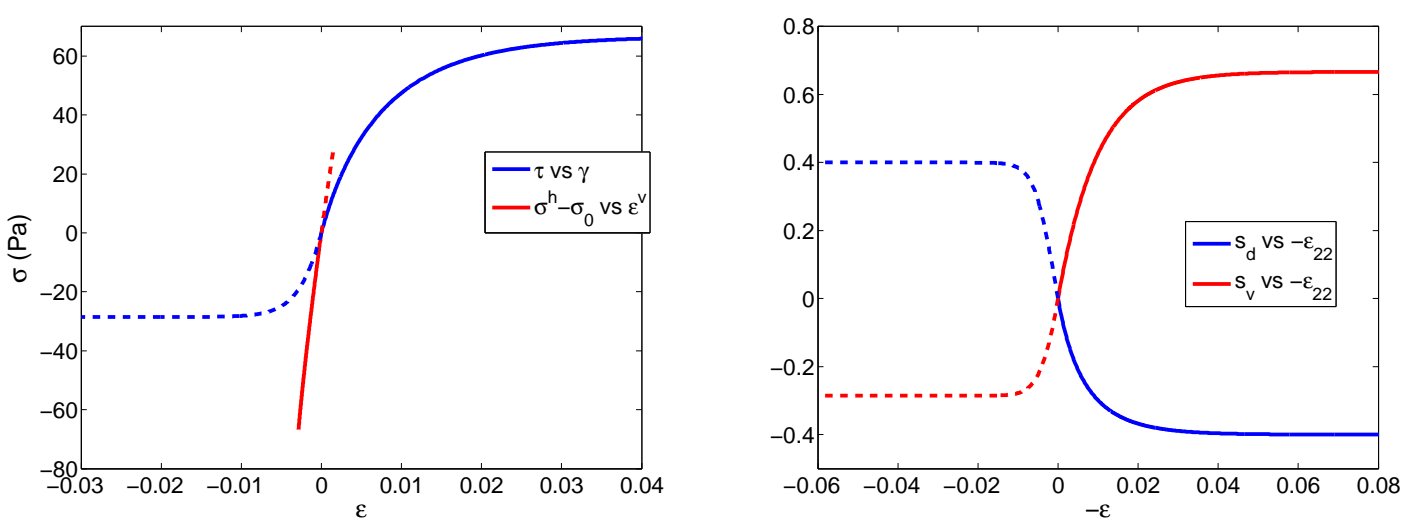

Figure 10: The development of shear and isotropic stresses (Left) and of the stress ratios (Right), during the biaxial deformation of the system, mode 3, with vertically prescribed compression (solid lines) or extension (dashed lines) and prescribed constant horizontal stress, $\sigma_{0}=-100 \mathrm{~Pa}$, for evolving $A$, starting from $A_{0}=0$, and maximal stress-anisotropy, using the standard parameters as given in the main text, together with $\beta_{A}=82, A^{m}=2 B / 5$, and $s_{d}^{m}=0.4$.

The isotropic strain, for example (data not shown), indicates the initial dilation and then saturates at around $1-2 \%$ of vertical strain, faster than under vertical compression, while the deviatoric strain decreases continuously with increasing slope (during the initial deformation) and constant slope later on. The continuously decreasing shear strain is due to the horizontal compression and vertical extension, which both enter $\gamma$ negatively. Thus the strains reach their limit states faster under vertical extension than under compression, and the limit states are different, dependent on the direction of strain, since the limit anisotropy is different (in sign).

When plotting the isotropic stress against isotropic strain and the deviatoric stress against deviatoric strain in Fig. 10 (Left), for extension (positive $\varepsilon$ ), volume increase is accompanied by an increase in isotropic stress, but the magnitude is smaller than for compression (negative $\varepsilon$ ). The deviatoric stress becomes negative (for extension) and saturates at a smaller magnitude and at smaller deviatoric strain than under compression.

In Fig. 10 (Right), the non-dimensional shear stress ratio, $s_{d}$, under vertical extension, increases and saturates at $s_{d}^{\max }=0.4$, as prescribed. Note that the saturation regime is reached faster for extension (after about 1\% of strain) than for compression (after about $5 \%$ of strain). The isotropic stress ratio also saturates, faster for extension, at a negative value $s_{v}^{\max }=-\left(1 / s_{d}^{\max }+1\right)^{-1}=-\frac{2}{7}$, which was not prescribed, but follows from the critical limit state parameters.

Best Practice Tip 4.2: The compression mode 3 leads to saturated shear stress, like the extension mode 3, where the former is considerably slower than the latter (with about 5\% of strain vs. 1-2\% to reach saturation). Starting from an initial isotropic system $\left(A_{0}=0\right)$, the equivalent moduli (initial slope of the stress strain curve) are identical (note the different range of isotropic stress under compression and extension). The anisotropy develops with the same rate and reaches saturation at about $7 \%$ of strain for the chosen model parameters.

These observations and predictions, based on the simplified version of the model, wait for experimental validation. If the experimental results show different behavior, this will show where to improve the model. 


\section{Summary and Conclusion}

In the biaxial system - where the eigen-vectors of all tensors are either horizontal or vertical - a new constitutive model, as based on DEM simulations in Ref. [18,22], is presented in Eqs. (38), (39), and (40). It involves the hydrostatic and deviatoric stresses, together with one anisotropy modulus that evolves during deviatoric deformations of the system (independently from the stress evolution, due to the constant parameters used here - for the sake of simplicity) and thus represents a history/memory parameter.

The five macroscopic field quantities are the hydrostatic stress, the shear stress, the anisotropy modulus, the isotropic strain and the shear strain. The model involves only three moduli: the classical bulk modulus $B$, a shear modulus, $G$, and the anisotropy modulus $A$, whose sign indicates the direction of anisotropy in the present formulation. ${ }^{8}$ Due to the anisotropy, $A$, the model involves a cross coupling of the two types of strains and stresses, namely isotropic and shear (deviatoric). As opposed to isotropic materials, shear strain can cause dilatation (compaction) and hence larger (smaller) compressive stresses. Similarly, a pure isotropic strain can cause shear stresses and thus shear deformation in the system. The shear stress evolution with shear strain is proportional to $2 G$, while the anisotropy grows proportional to shear strain and the rate parameter $\beta_{A}$.

The model also leads to a critical state regime, where the stresses and the anisotropy modulus do not change anymore, given constant $B$ and $G .{ }^{9}$ The critical state is described by the maximal anisotropy $A^{m}$ and the maximal deviatoric stress ratio $s_{d}^{m}$, where the latter is equivalent to a macroscopic friction coefficient in Mohr-Coulomb type constitutive relations, and thus represents a yield-stress limit.

\subsection{Micro-Macro Mechanics and Constitutive Relations}

The flow of granular materials is controlled by the micro-mechanics of the disordered structure of the material. On the macroscopic level of description, one history parameter is introduced that accounts for the micro-mechanical anisotropy evolution of the system. This single new parameter is the only extension as compared to classical continuum theories. Furthermore, all tensors are split into their scalar components, which is possible only in the bi-axial system. In more general situations, the tensors can be split into their (scalar) moduli and a non-dimensional unit-deviator ("director") that involves the orientation of the tensor eigen-system. In two dimensions (2D) this is particularly simple, since each tensor direction is uniquely defined by one angle.

In contrast to (some, piece-wise linear) elasto-plastic models, the present evolution of stress and anisotropy is smooth: both $s_{d}$ and $A$ approach their critical state regime exponentially fast, which means a linear regime for small strain and a critical state regime for large strain, without any discontinuities of the derivatives. Strain reversal, on the other hand, leads to a non-linear stress evolution - again due to the presence of the anisotropy - with different loading and un-loading stiffnesses, as in hypo-plasticity type models.

Note that the present model does not show softening - softening was not inserted, but shows up as an artefact when, e.g., the inconsistent variant of the model is used (data not shown). Disregarding softening is on purpose, since the model is only locally valid (or for homogeneous systems), whereas softening and other effects are (to our knowledge) observed for inhomogeneous systems. Softening - in our philosophy - should NOT be inserted into a (simple) local model, since it is expected to be the result of the collective behavior of the material in a particular experiment with its boundary conditions.

The condition when the system becomes instable has also been derived analytically and is fulfilled

\footnotetext{
${ }^{8}$ Future formulations will take into account arbitrary orientations of stress, anisotropy and strain.

${ }^{9}$ The latter is a simplification, where evolution equations for $B$ and $G$ will be introduced elsewhere [39]. Their change is often smaller than the variation of the new modulus, $A[18]$.
} 
automatically in the critical state regime: The existence of anisotropy in the model causes instability when it becomes larger than a certain value that is a function of the bulk and shear moduli. However, interestingly, such large anisotropy was never observed in DEM simulations, which leaves the question about the particulate origin of the instability open.

\subsection{From Constant to Evolving Anisotropy}

To better understand the model, a series of simulations has been performed for special cases: Some analytical predictions can be made for constant anisotropy in the system. As expected, linear relations between strains and stresses are observed, in line with the coupling via the (constant) anisotropy mentioned above. The constant anisotropy also reflects the system behavior with evolving anisotropy for very small strains. For larger strains the non-linear behavior sets in with a particular interplay between isotropic and deviatoric components, which are cross-coupled by the anisotropy. The evolving anisotropy of the structure, $A$, and of the stress ratio, $s_{d}$, both with a maximum, lead to non-linear effects as the critical state regime and the change of stiffness under strain-reversal.

\subsection{Future Work}

The next step is the formulation of the model for arbitrary orientations of the stress-, strainand anisotropy-tensors, but keeping the number of parameters fixed. This is a requirement for a possible Finite Element Method implementation of the model, in order to study arbitrary boundary conditions other than bi-axial systems.

After this is achieved, the model can be generalized to three dimensions (3D), where (at least) one additional anisotropy parameter (one new contribution to the anisotropy tensor with different orientation) is expected to be present for arbitrary deformation histories. Otherwise, the spirit of the model will not be different in 3D.

\section{Acknowledgments}

Helpful discussions with J. Goddard, D. Krijgsman, M. Liu, V. Magnanimo, S. McNamara, A. Singh, S. Srivastava, H. Steeb, J. Sun, S. Sundaresan, J. Tejchman and W. Wu are gratefully acknowledged. This work was financially supported by MicroNed grant 4-A-2, by the research programme of the "Stichting voor Fundamenteel Onderzoek der Materie" (FOM), which is financially supported by the "Nederlandse Organisatie voor Wetenschappelijk Onderzoek" (NWO), and by an NWO-STW VICI grant.

\section{References}

[1] H. M. Jaeger, S. R. Nagel, and R. P. Behringer. Granular solids, liquids, and gases. Rev. Mod. Phys., 68(4):1259-1273, 1996.

[2] P. G. de Gennes. Granular matter: a tentative view. Reviews of Modern Physics, 71(2):374$382,1999$.

[3] I. Vardoulakis and J. Sulem. Bifurcation analysis in geomechanics. Chapman \& Hall, London, 1995.

[4] A. J. Liu and S. R. Nagel. Nonlinear dynamics - Jamming is not just cool any more. Nature, 396(6706), 1998. 
[5] C. S. O'Hern, S. A. Langer, A. J. Liu, and S. R. Nagel. Random packings of frictionless particles. Phys. Rev. Lett., 88(7), 2002.

[6] T. S. Majmudar, M. Sperl, S. Luding, and R. P. Behringer. Jamming transition in granular systems. Phys. Rev. Lett., 98(5):058001, 2007.

[7] Stefan Luding. Cohesive, frictional powders: contact models for tension. Granular matter, $10(4), 2008$.

[8] Pierre-Emmanuel Peyneau and Jean-Noel Roux. Solidlike behavior and anisotropy in rigid frictionless bead assemblies. Phys. Rev. E, 78(4, Part 1), 2008.

[9] J. Zhang, T. S. Majmudar, A. Tordesillas, and R. P. Behringer. Statistical properties of a 2d granular material subjected to cyclic shear. Granular Matter, 12:159-172, 2010.

[10] L. R. Aarons, J. Sun, and S. Sundaresan. Unsteady shear of dense assemblies of cohesive granular materials under constant volume conditions. Ind. Eng. Chem. Res., 49:5153-5165, 2010.

[11] J. Sun and S. Sundaresan. A plasticity model with microstructure evolution for quasi-static granular flows. J. Fluid Mech., submitted, 2011.

[12] F. Radjai, D. E. Wolf, M. Jean, and J. J. Moreau. Bimodal character of stress transmission in granular packings. Phys. Rev. Lett., 80(1):61-64, 1998.

[13] F. Radjai, S. Roux, and J. J. Moreau. Contact forces in a granular packing. Chaos, 9(3):544, 1999.

[14] F. Radjai and S. Roux. Turbulentlike fluctuations in quasistatic flow of granular media. Phys. Rev. Lett., 89(6):064302-1, 2002.

[15] S. D. C. Walsh and A. Tordesillas. A thermomechanical approach to the development of micropolar constitutive models of granular media. Act. Mech., 167:145-169, 2004.

[16] F. Alonso-Marroquin, S. Luding, H. J. Herrmann, and I. Vardoulakis. Role of anisotropy in the elastoplastic response of a polygonal packing. Phys. Rev. E, 71, 2005.

[17] P. A. Vermeer, S. Diebels, W. Ehlers, H. J. Herrmann, S. Luding, and E. Ramm, editors. Continuous and Discontinuous Modelling of Cohesive Frictional Materials, Berlin, 2001. Springer.

[18] S. Luding. Anisotropy in cohesive, frictional granular media. J. Phys.: Condens. Matter, 17:S2623-S2640, 2005.

[19] M. Lätzel, S. Luding, and H. J. Herrmann. Macroscopic material properties from quasi-static, microscopic simulations of a two-dimensional shear-cell. Granular Matter, 2(3):123-135, 2000.

[20] M. Lätzel, S. Luding, and H. J. Herrmann. From discontinuous models towards a continuum description. In P. A. Vermeer, S. Diebels, W. Ehlers, H. J. Herrmann, S. Luding, and E. Ramm, editors, Continuous and Discontinuous Modelling of Cohesive Frictional Materials, pages 215230, Berlin, 2001. Springer.

[21] S. Luding, M. Lätzel, W. Volk, S. Diebels, and H. J. Herrmann. From discrete element simulations to a continuum model. Comp. Meth. Appl. Mech. Engng., 191:21-28, 2001.

[22] S. Luding. Micro-macro models for anisotropic granular media. In P. A. Vermeer, W. Ehlers, H. J. Herrmann, and E. Ramm, editors, Modelling of Cohesive-Frictional Materials, pages 195-206, Leiden, Netherlands, 2004. Balkema.

[23] Stefan Luding. Constitutive relations for the shear band evolution in granular matter under large strain. Particuology, 6(6), 501-505 2008.

[24] I. Goldhirsch. Stress, stress asymmetry and couple stress: from discrete particles to continuous fields. Granular Matter, 12:239-252, 2010. 
[25] J. Goddard. Granular hypoplasticity with cosserat effects. In J. Goddard, P. Giovine, and J. T. Jenkins, editors, IUTAM-ISIMM Symposium on Mathematical Modeling and Physical Instances of Granular Flows, pages 323-332, Reggio Calabria (Italy), 14.18 September 2009, 2010. AIP.

[26] Hans Muhlhaus, Louis Moresi, Lutz Gross, and Joseph Grotowski. The influence of noncoaxiality on shear banding in viscous-plastic materials. Granular Matter, 12:229-238, 2010.

[27] J. Sun and S. Sundaresan. A plasticity model with microstructure evolution for quasi-static granular flows In J. Goddard, P. Giovine, and J. T. Jenkins, editors, IUTAM-ISIMM Symposium on Mathematical Modeling and Physical Instances of Granular Flows, pages 280-289, Reggio Calabria (Italy), 14.18 September 2009, 2010. AIP.

[28] J. P. Bardet and I. Vardoulakis. The asymmetry of stress in granular media. Int. J. of Solids and Structures, 38(2):353-367, 2001.

[29] I. Vardoulakis. Shear-banding and liquefaction in granular materials on the basis of a Cosserat continuum theory. Ingenieur-Archiv, 59:106-113, 1989.

[30] J. Tejchman and W. Wu. Modeling of textural anisotropy in granular materials with stochastic micro-polar hypoplasticity. Int. J. of Non-Linear Mechanics, 42:882-894, 2007.

[31] S. Luding. Macroscopic stress from dynamic, rotating granular media. In J. Goddard, P. Giovine, and J. T. Jenkins, editors, IUTAM-ISIMM Symposium on Mathematical Modeling and Physical Instances of Granular Flows, pages 208-213, Reggio Calabria (Italy), 14.18 September 2009, 2010. AIP.

[32] J. D. Goddard. Parametric hypoplasticity as continuum model for granular media: from stokesium to mohr-coulombium and beyond. Granular Matter, 12:145-150, 2010.

[33] Y. Jiang and M. Liu. Granular solid hydrodynamics. Granular Matter, 11:139-156, 2009.

[34] S. Mahle, Y. Jiang, and M. Liu. The critical state as a steady-state solution of granular solid hydrodynamics. arxiv.org/abs/1006.5131v3, 2010. preprint.

[35] F. Nicot and F. Darve. On the elastic and plastic strain decomposition in granular materials. Granular Matter, 8:221-237, 2006.

[36] V. Magnanimo and S. Luding. A local constitutive model with anisotropy for ratcheting under 2d biaxial isobaric deformation. Granular Matter, 13(3):???, 2011. DOI: ??? submitted.

[37] D. O. Brush and B. O. Almroth. Buckling of Bars, Plates, and Shells. McGraw Hill, 1975.

[38] S. Luding and F. Alonso-Marroquin. The critical-state yield stress (termination locus) of adhesive powders from a single numerical experiment. Granular Matter, 13(2):109-119, 2011. DOI: $10.1007 / \mathrm{s} 10035-010-0241-4$.

[39] F. Göncü, O. Duran, and S. Luding. Constitutive relations for the isotropic deformation of frictionless packings of polydisperse spheres. C. R. Mecanique, 338 (10-11):570-586, 2010. 